\title{
Generic Initial Ideals and Distractions
}

\author{
A.M. Bigatti, A. Conca, L. Robbiano *
}

\begin{abstract}
The generic initial ideals of a given ideal are rather recent invariants. Not much is known about these objects, and it turns out to be very difficult to compute them. The main purpose of this paper is to study the behaviour of generic initial ideals with respect to the operation of taking distractions. Theorem 4.3 is our main result. It states that the DegRevLex-generic initial ideal of the distraction of a strongly stable ideal is the ideal itself. In proving this fact we develop some new results related to distractions, stable and strongly stable ideals. We draw some geometric conclusions for ideals of points.
\end{abstract}

\section{Introduction}

What song the Syrens sang, or what name Achilles assumed when he hid himself among women, although puzzling questions are not beyond all conjecture.

Sir Thomas Browne, Urn-Burial (quoting the quote of E. A. Poe to "The Murders in the Rue Morgue")

Everything related to the present paper started some time ago when we were playing with CoCoA. One should never underestimate the importance of playing, even in Mathematics; fortunately nowadays we have wonderful computer algebra toys. They are a sort of scientific version of videogames which for instance allow us to continually explore the hidden secrets of polynomial rings.

This paper provides another consequence of this new working behaviour of many mathematicians. The story starts with the three of us playing with generic initial ideals. First of all, it should be said that when computing generic initial ideals, one's trust in the answers lies not only on the reliability of the computer algebra system, but also on a more subtle belief. Namely, even for very easy examples, it turns out that a true computation of generic initial ideals is infeasible. When we say a true computation, we mean a computation with a

*AMS Classification: 13C99, 13P10 
linear change of coordinates achieved with a truly generic matrix, i.e. a matrix whose entries are distinct indeterminates.

What do we usually do instead? We perform a linear change of coordinates with a random matrix, and trust the answer. In practice, we believe that a random choice of a point in a projective space does not hit a given hypersurface. Of course the probability of hitting such a hypersurface is zero, but we might be extremely unlucky. It is like thinking of a natural number and asking someone else to guess it: the probability of success is zero, but ...

A good mathematician could argue that examples computed in this way are not acceptable, and from that point of view this paper brings good news, since we are able to produce a mathematical proof which leads to the identification of an interesting class of generic initial ideals (see for instance Theorem 4.3, Theorem 4.5, and Corollary 4.10).

So, let us have a closer look at the content, and start off by recalling two known results about generic initial ideals.

The first result (see Proposition 1.8) says that if $I$ is a Borel-fixed ideal (see Definition 1.2) in the polynomial ring $P=K\left[x_{1}, \ldots, x_{n}\right]$, and $\sigma$ is a term ordering, then $\operatorname{gin}_{\sigma}(I)=I$.

The second result (see Theorem 1.13)) states that if $I$ is a homogeneous ideal in $P, h$ a generic linear form, and $\sigma$ a term ordering of $x_{n}$-DegRev type (see Definition 1.11), then we have $\operatorname{gin}_{\sigma_{\hat{x}_{n}}}\left(I_{h}\right)=\left(\operatorname{gin}_{\sigma}(I)\right)_{x_{n}}$. Roughly speaking, the equality can be expressed by saying that if $\sigma$ is a term ordering of $x_{n}$-DegRev type, then taking the $\operatorname{gin}_{\sigma_{\hat{x}_{n}}}$ of a generic hyperplane section of $I$, is like taking the $x_{n}$-hyperplane section of the gin ${ }_{\sigma}$ of $I$.

While playing with CoCoA, we noticed that for a special kind of ideal, namely some ideals of distractions (see Definitions 2.1 and 2.2) stemming from strongly stable ideals (see Definition 1.3), an even more interesting equality holds, namely $\operatorname{gin}_{{\widehat{\hat{x}_{n}}}_{\widehat{x}_{n}}}\left(I_{x_{n}}\right)=\left(\operatorname{gin}_{\sigma}(I)\right)_{x_{n}}$, provided $x_{n}$ does not divide zero modulo $I$. This fact was purely empirical, but more than enough to conjecture the validity of the equality in the case of distractions of strongly stable ideals and $x_{n}$-DegRev type orderings.

One might ask why we were considering strongly stable ideals. The motivation comes from the fact that these ideals are Borel-fixed (see Proposition 1.5). And we have already mentioned the fact that if $I$ is Borel-fixed, then $\operatorname{gin}_{\sigma}(I)=I$ which might suggest other forms of stability for this type of ideal. The turning point happened when CoCoA completed the following session.

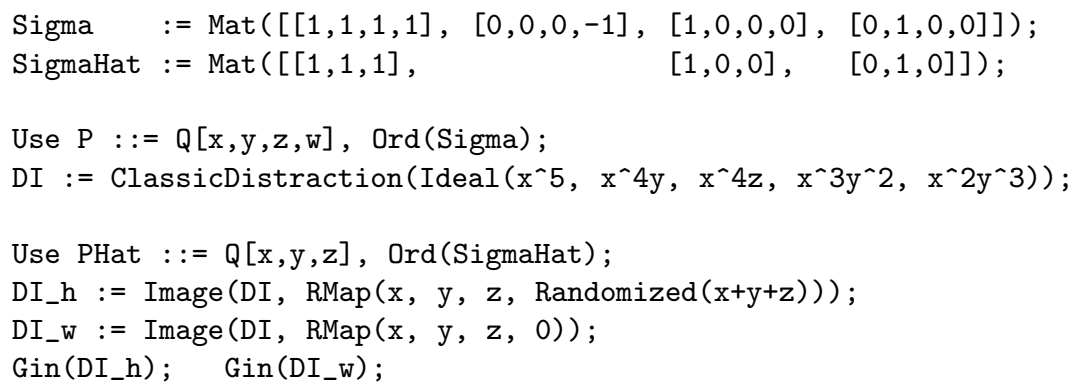




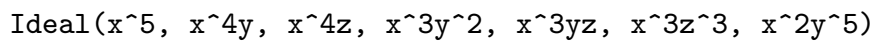

Ideal $\left(x^{\wedge} 5, x^{\wedge} 4 y, x^{\wedge} 4 z, x^{\wedge} 3 y^{\wedge} 2, x^{\wedge} 2 y^{\wedge} 3\right)$

This was a counterexample to our conjecture. Nevertheless we were unable to find any counterexample to the more restricted conjecture that the equality $\operatorname{gin}_{\sigma_{\hat{x}_{n}}}\left(I_{x_{n}}\right)=\left(\operatorname{gin}_{\sigma}(I)\right)_{x_{n}}=I_{x_{n}}$ is true for the same kind of ideals of distractions as above, and $\sigma=$ DegRevLex. A huge amount of experimental evidence was accumulated. Fortunately, the solution, although non trivial, turned out to be more reachable than discovering what song the Syrens sang, and we were indeed able to prove the main Theorem 4.2 , from which the validity of the conjecture stems as an easy consequence, since Theorem 4.3 shows that $\operatorname{gin}_{\mathrm{drl}}\left(D_{\mathcal{L}}(I)\right)=I$ for every strongly stable ideal $I$.

At that point one might be lead to conjecture that $\operatorname{gin}_{\mathrm{drl}}\left(D_{\mathcal{L}}(I)\right)=\operatorname{gin}_{\mathrm{drl}}(I)$ for every monomial ideal. This is not true even for stable ideals (see the second part of Example 4.6). However, it is true for special monomial ideals which include principal stable ideals (see Theorem 4.5).

The road to the proofs had to be paved using some preparatory material, and to this end we devote Sections 1, 2 and 3. In particular, Section 2 develops some elements of the theory of distractions. For instance, we give a direct proof that monomial ideals and their distractions not only share the same Hilbert function (Corollary 2.10), but also share the same standard Betti numbers (Theorem 2.19 and Corollary 2.20).

Distractions can be also described as specializations of polarizations of monomial ideals. They were (essentially) introduced by Hartshorne in his proof of the connectedness of the Hilbert scheme [H66], and since then they have been used, described, and rediscovered by many authors. In this context let us mention the work of Pardue [P94, P96] and the work of Migliore and Nagel [MN00].

Finally, we point out an interesting application of our main results. By using suitable distractions of a strongly stable monomial ideal $I$, one can construct schemes of rational points whose gin with respect to DegRevLex is $I$ itself (see Corollary 4.10). In that case you do not have to rely on luck to claim that you know the gin, you have a proof! 


\section{Preliminaries}

In this section we state the main preliminary facts. Although all the results discussed here are known, the proofs given in the literature are not, in general, very accurate. For the sake of completeness we prefer to include some of them here.

In the following we let $P=K\left[x_{1}, \ldots, x_{n}\right]$ be a polynomial ring with $n$ indeterminates over an infinite field $K$. We also assume that it is standard graded, meaning that its grading satisfies $\operatorname{deg}(c)=0$ for every $c \in K \backslash\{0\}$, and $\operatorname{deg}\left(x_{i}\right)=1$ for every $i=1, \ldots, n$. In other words, the degree matrix of the grading is $(1,1, \ldots, 1)$ (see [KR04] for more details on gradings defined by matrices).

The next definition introduces the notion of $\operatorname{in}_{\sigma}(f)$ for a given term ordering $\sigma$, and a non-zero polynomial $f$. The full theory can be read for instance in [KR00]. It should be noted that here we use $\operatorname{in}_{\sigma}(f)$ instead of $\operatorname{LT}_{\sigma}(f)$, and the reason is that the notation $\operatorname{in}(I)$ is compatible with $\operatorname{gin}(I)$, which will be the main object under investigation in this paper (gLT does not look too good, it does not sound too good either!).

Definition 1.1. Let $I$ be an ideal in $P$ and let $\sigma$ be a term ordering on $\mathbb{T}^{n}$, the monoid of power products in $x_{1}, \ldots, x_{n}$. We denote by $\operatorname{in}_{\sigma}(I)$ the monomial ideal generated by $\left\{\operatorname{in}_{\sigma}(f) \mid f \in I \backslash\{0\}\right\}$ and call it the initial ideal of $I$, or the leading term ideal of $I$.

Throughout the paper we are always assuming that $\sigma$ is a degree-compatible term ordering such that $x_{1}>_{\sigma} x_{2}>_{\sigma} \cdots>_{\sigma} x_{n}$. The only exception to this assumption is made when we talk about term orderings of $x_{i}$-DegRev type (see Definition 1.11, Proposition 1.12 and Theorem 1.13).

Let us consider an automorphism $\operatorname{Aut}_{K}\left(P_{1}\right)$ on the $K$-vector space $P_{1}$. If we choose $x_{1}, \ldots, x_{n}$ as a basis of $P_{1}$, we may as usual describe the automorphism via a matrix $\mathfrak{g}=\left(g_{i j}\right) \in \mathrm{GL}(n, K)$ with entries in $K$.

If $\gamma \in \operatorname{Aut}_{K}\left(P_{1}\right)$ is represented by $\mathfrak{g}$, then $\gamma\left(x_{j}\right)=\sum_{i=1}^{n} g_{i j} x_{i}$. Therefore we have

$$
\left(\gamma\left(x_{1}\right), \ldots, \gamma\left(x_{n}\right)\right)=\left(x_{1}, \ldots, x_{n}\right) \cdot \mathfrak{g}
$$

where the "." indicates the usual row-by-column product of matrices. The action of $\mathfrak{g}$ on $P_{1}$ extends to $P$, so that it makes sense to consider objects like $\mathfrak{g}(f)$ and $\mathfrak{g}(I)$.

We can parametrize $\operatorname{Aut}_{K}\left(P_{1}\right)$ with $\mathbb{A}_{K}^{n^{2}} \backslash H$, where $H$ is the hypersurface defined by the vanishing of the determinant of a matrix whose entries are $n^{2}$ distinct indeterminates. Having assumed $K$ to be infinite, it make sense to speak of a generic matrix, likewise a generic automorphism.

Definition 1.2. The subgroup $B$ of $\mathrm{GL}(n, K)$ of the upper triangular matrices is called the Borel subgroup. If $I$ is an ideal in $P$, we say that $I$ is Borelfixed if $\mathfrak{g}(I)=I$ for every $\mathfrak{g} \in B$. 
Definition 1.3. A subvector space of $P$ is called a monomial vector space if it has a basis of power products. An ideal in $P$ which is a monomial vector space is called a monomial ideal.

If $t \in \mathbb{T}^{n}$, and $r$ is the biggest integer such that $x_{r} \mid t$, we call $r$ the maximum index of $t$ and denote it by $\mathrm{m}(t)$. Let $T$ be a finite set of power products, and $V$ the monomial vector space spanned by $T$. The set $T$ and the vector space $V$ are called stable if $T$ satisfies the following property: for every power product $t \in V$ and every $i \leq \mathrm{m}(t)$, then $x_{i} \frac{t}{x_{\mathrm{m}(t)}} \in V$.

The set $T$ and the vector space $V$ are called strongly stable if $T$ satisfies the following property: for every power product $t \in V$ such that $x_{j} \mid t$ and every $i \leq j$, then $x_{i} \frac{t}{x_{j}} \in V$.

REMARK 1.4. It is easy to see that if an ideal $I$ is Borel-fixed, then it is necessarily a monomial ideal.

Proposition 1.5 Let $K$ be a field, and let $I$ be a monomial ideal in the polynomial ring $K\left[x_{1}, \ldots, x_{n}\right]$. Consider the following conditions.

a) The ideal $I$ is Borel-fixed

b) The ideal $I$ is strongly stable

Then $b) \Rightarrow a)$. Moreover, if $\operatorname{char}(K)=0$ they are equivalent.

Proof. See for instance Proposition 1.25 in [G96].

We are ready to recall a celebrated theorem. It was proved by Galligo in characteristic zero, and then generalized by Bayer and Stillman to every characteristic.

\section{Theorem 1.6 (Galligo-Bayer-Stillman)}

Let $K$ be an infinite field, let $\sigma$ be a term ordering on $\mathbb{T}^{n}$, and let $I$ be a homogeneous ideal in $P$. The following facts hold true.

a) The ideal $\operatorname{in}_{\sigma}(\mathfrak{g}(I))$ is constant for a generic $\mathfrak{g}$.

b) The ideal $\operatorname{in}_{\sigma}(\mathfrak{g}(I))$ is Borel-fixed for a generic $\mathfrak{g}$.

Proof. See for instance [G96] p. 26.

This theorem allows us to give the following definition.

Definition 1.7. Let $\sigma$ be a term ordering on $\mathbb{T}^{n}$, and let $I$ be a homogeneous ideal in $P$. We denote by $\operatorname{gin}_{\sigma}(I)$ the Borel-fixed monomial ideal which is equal to $\operatorname{in}_{\sigma}(\mathfrak{g}(I))$ for a generic $\mathfrak{g}$, as prescribed by the theorem. We call it the generic initial ideal of $I$ with respect to $\sigma$, or the $\sigma$-generic initial ideal of $I$. Using this terminology, part b) of the theorem can be stated by saying that $\operatorname{gin}_{\sigma}(I)$ is Borel-fixed, and hence strongly stable in characteristic 0 by Proposition 1.5. 


\section{Proposition 1.8 (Gin and Borel-Fixed Ideals)}

Let $K$ be an infinite field, let $\sigma$ be a term ordering on $\mathbb{T}^{n}$, and let $I$ be a Borel-fixed ideal in $P$. Then $\operatorname{gin}_{\sigma}(I)=I$.

ProOf. It is a well-known fact, and here we give only a hint. Recall that if $\gamma \in \operatorname{Aut}_{K}\left(P_{1}\right)$ is represented by $\mathfrak{g}$, then $\left(\gamma\left(x_{1}\right), \ldots, \gamma\left(x_{n}\right)\right)=\left(x_{1}, \ldots, x_{n}\right) \cdot \mathfrak{g}$. If $\gamma$ is generic we can decompose $\mathfrak{g}$ as the product of a lower triangular times an upper triangular matrix. Then we observe that the action of the lower triangular matrix on a polynomial does not change its leading term. Moreover $I$ is closed under the action of the upper triangular matrix since $I$ Borel-fixed. The conclusion follows from the fact that $\operatorname{gin}_{\sigma}(I)$ and $I$ have the same Hilbert function.

EXAMPLE 1.9. The following is an easy example of a stable ideal which is not strongly stable. In the polynomial ring $K\left[x_{1}, x_{2}, x_{3}\right]$, consider the following ideal $I=\left(x_{1}^{2}, x_{1} x_{2}, x_{2}^{2}, \underline{x_{2} x_{3}}\right)$. One can check (with CoCoA) that $\operatorname{gin}_{\mathrm{drl}}(I)=$ $\left(x_{1}^{2}, x_{1} x_{2}, x_{2}^{2}, \underline{x_{1} x_{3}}\right)$.

At this point, we want to discuss what happens when we consider hyperplane sections. Let $i \in\{1, \ldots, n\}$, let $h=\sum_{j=1}^{n} h_{j} x_{j} \in P_{1}$ be a linear form such that $h_{i} \neq 0$, and call $\hat{P}=K\left[x_{1}, \ldots, x_{i-1}, x_{i+1}, \ldots, x_{n}\right]$. The homomorphism $\varphi: P \longrightarrow \hat{P}$ defined by $\varphi\left(x_{j}\right)=x_{j}$ for $j \neq i, \varphi\left(x_{i}\right)=-\frac{1}{h_{i}}\left(\sum_{j \neq i} h_{j} x_{j}\right)$, induces an isomorphism between $P /(h)$ and $\hat{P}$, which allows us to give the following definition.

Definition 1.10. Given a polynomial $f \in P$, we call $f_{h}$ the polynomial $\varphi(f)$ in $\hat{P}$. More generally, given a homogeneous ideal $I$ in $P$, we call $I_{h}$ the ideal $\varphi(I)$ in $\hat{P}$. It is named the $h$-hyperplane section ideal or simply the $h$-hyperplane section of $I$.

It should be observed that Definition 1.10 is the correct algebraic definition, but it does not correspond to the geometric notion of a hyperplane section. The reason is that, even if $I$ is saturated, $I_{h}$ need not to be such.

Finally, we need another essential tool, namely the notion of term ordering of $x_{i}$-DegRev type. It is an important class of term orderings which includes the so called DegRevLex ordering (see [KR04] for a through treatment of this topic).

Definition 1.11. Let $i \in\{1, \ldots, n\}$. We say that a term ordering $\sigma$ on $\mathbb{T}^{n}$ is of $x_{i}$-DegRev type, if it satisfies the following conditions:

a) The ordering $\sigma$ is degree-compatible.

b) Given $t, t^{\prime} \in \mathbb{T}^{n}$, such that $\operatorname{deg}(t)=\operatorname{deg}\left(t^{\prime}\right)$ and $\log _{x_{i}}(t)<\log _{x_{i}}\left(t^{\prime}\right)$, we have $t>_{\sigma} t^{\prime}$. 
We observe that term orderings of $x_{i}$-DegRev type exist. Namely, such an ordering can be obtained as $\operatorname{Ord}(V)$, where $V$ is a matrix whose first two rows are $\left(\begin{array}{ccccc}1 & \cdots & 1 & \cdots & 1 \\ 0 & \cdots & -1 & \cdots & 0\end{array}\right)$.

The main result about term orderings of $x_{i}$-DegRev type is contained in the following proposition. We use the notation $\sigma_{\hat{x}_{i}}$ to indicate the restriction of the term ordering $\sigma$ to the monoid of power products in the indeterminates $x_{1}, \ldots, x_{i-1}, x_{i+1}, \ldots, x_{n}$.

Proposition 1.12 Let $I$ be a homogeneous ideal in $P$, let $i \in\{1, \ldots, n\}$, let $\sigma$ be a term ordering of $x_{i}$-DegRev type on $\mathbb{T}^{n}$, and let $G=\left\{g_{1}, \ldots, g_{s}\right\}$ be a homogeneous $\sigma$-Gröbner basis of $I$.

a) The set $\left\{\left(g_{1}\right)_{x_{i}}, \ldots,\left(g_{s}\right)_{x_{i}}\right\} \backslash\{0\}$ is a homogeneous $\sigma_{\hat{x}_{i}}$-Gröbner basis of the ideal $I_{x_{i}}$.

b) We have $\left(\operatorname{in}_{\sigma}(I)\right)_{x_{i}}=\operatorname{in}_{\sigma_{\hat{x}_{i}}}\left(I_{x_{i}}\right)$.

c) We have $\operatorname{in}_{\sigma}(I)+\left(x_{i}\right)=\operatorname{in}_{\sigma}\left(I+\left(x_{i}\right)\right)$.

Proof. The proof is easy. A full discussion and generalization can be found in [KR04].

Now we are ready to state an important theorem in the theory of generic initial ideals. Given a homogeneous ideal $I$, it compares the $\sigma_{\hat{x}_{i}}$-generic initial ideal of the generic hyperplane section of $I$, with the $x_{i}$-hyperplane section of the $\sigma$-generic initial ideal of $I$. We were unable to find any good proof in the literature, so we decided to include one here.

\section{Theorem 1.13 (Gin and Hyperplane Sections)}

Let $I$ be a homogeneous ideal in $P$, let $h \in P_{1}$ be a generic linear form, let $i \in\{1, \ldots, n\}$, and let $\sigma$ be a term ordering of $x_{i}$-DegRev type. Then we have the equality

$$
\operatorname{gin}_{\sigma_{\widehat{x}_{i}}}\left(I_{h}\right)=\left(\operatorname{gin}_{\sigma}(I)\right)_{x_{i}}
$$

of ideals in $K\left[x_{1}, \ldots, x_{i-1}, x_{i+1}, \ldots, x_{n}\right]$.

Proof. To simplify the presentation, we give the proof for $i=n$, since the general case is a straightforward generalization. The linear form $h=\sum_{i=1}^{n} h_{i} x_{i}$ is generic, hence $h_{n} \neq 0$. We consider a generic matrix $\mathfrak{g} \in \mathrm{GL}(n-1, K)$, and construct another matrix $\mathfrak{g}^{\prime}=\left(\begin{array}{c|c}\mathfrak{g} & v^{\text {tr }} \\ \hline 0 & 0\end{array}\right)$, where $v=\left(-\frac{h_{1}}{h_{n}}, \cdots,-\frac{h_{n-1}}{h_{n}}\right)$. We observe that $v$ is a generic vector in $K^{n-1}$.

It is clear that

$$
\left(x_{1}, \ldots, x_{n-1}, x_{n}\right) \cdot \mathfrak{g}^{\prime}=\left(\left(x_{1}, \ldots, x_{n-1}\right) \cdot \mathfrak{g} \mid-\frac{1}{h_{n}}\left(\sum_{i=1}^{n-1} h_{i} x_{i}\right)\right)
$$

hence we get the first equalities

$$
\operatorname{gin}_{\sigma_{\hat{x}_{n}}}\left(I_{h}\right)=\operatorname{in}_{\sigma_{\hat{x}_{n}}}\left(\mathfrak{g}\left(I_{h}\right)\right)=\operatorname{in}_{\sigma_{\hat{x}_{n}}}\left(\mathfrak{g}^{\prime}(I)\right)
$$


Now, we consider a generic matrix $\mathfrak{G} \in G L(n, K)$, and get the equality $\operatorname{gin}_{\sigma}(I)=\operatorname{in}_{\sigma}(\mathfrak{G}(I))$ which implies the equality

$$
\left(\operatorname{gin}_{\sigma}(I)\right)_{x_{n}}=\left(\operatorname{in}_{\sigma}(\mathfrak{G}(I))\right)_{x_{n}}
$$

We may view $\mathfrak{G}$ as a block matrix in the following way $\mathfrak{G}=\left(\begin{array}{l|l}\mathfrak{g} & v^{\text {tr }} \\ \hline w & c\end{array}\right)$, where $v, w$ are generic vectors in $K^{n-1}$, and $c$ is a generic element in $K$. We obtain

$$
\left(\operatorname{in}_{\sigma}(\mathfrak{G}(I))\right)_{x_{n}}=\operatorname{in}_{\sigma_{\hat{x}_{n}}}\left(\mathfrak{G}(I)_{x_{n}}\right)
$$

by Proposition 1.12.b. Using equalities (1), (2) and (3) it suffices to prove that $\mathfrak{G}(I)_{x_{n}}=\mathfrak{g}^{\prime}(I)$ which follows immediately from the definition of $\mathfrak{G}$ and $\mathfrak{g}^{\prime}$. The proof is now complete.

EXAMPLE 1.14. With the following example we anticipate a theme which will be fully treated in the next sections. As we said in the introduction, we asked ourselves the following question. For ideals of distractions and a term ordering $\sigma$ of $x_{i}$-DegRev type, is it true that $\operatorname{gin}_{\sigma_{\hat{x}_{i}}}\left(I_{x_{i}}\right)=\left(\operatorname{gin}_{\sigma}(I)\right)_{x_{i}}$ ? In view of Theorem 1.13, the question could also be phrased in the following way. Is it true that $\operatorname{gin}_{\sigma_{\hat{x}_{i}}}\left(I_{x_{i}}\right)=\operatorname{gin}_{\sigma_{\hat{x}_{i}}}\left(I_{h}\right)$ ?

There is a $\mathrm{CoCoA}$ counterexample, even if we start with a strongly stable monomial ideal $I$. Namely, let $P=K[x, y, z, w]$, and let $I$ be the ideal in $P$ generated by $\left\{x^{5}, x^{4} y, x^{4} z, x^{3} y^{2}, x^{2} y^{3}\right\}$. Let $D(I)$ be the ideal in $P$ generated by $\{x(x-w)(x-2 w)(x-3 w)(x-4 w), x(x-w)(x-2 w)(x-3 w) y, x(x-$ $w)(x-2 w)(x-3 w) z, x(x-w)(x-2 w) y(y-w), x(x-w) y(y-w)(y-2 w)\}$.

Now let $\sigma=\operatorname{Ord}(W)$ be the term ordering defined by the matrix

$$
W=\left(\begin{array}{cccc}
1 & 1 & 1 & 1 \\
0 & 0 & 0 & -1 \\
1 & 0 & 0 & 0 \\
0 & 1 & 0 & 0
\end{array}\right)
$$

It is easy to see that $\sigma$ is of $w$-DegRev type, and a CoCoA computation yields the following results.

$$
\begin{aligned}
& \operatorname{gin}_{\sigma_{\hat{w}}}\left(D(I)_{h}\right)=\left(x^{5}, x^{4} y, x^{4} z, x^{3} y^{2}, x^{3} y z, x^{3} z^{3}, x^{2} y^{5}\right) \\
& \operatorname{gin}_{\sigma_{\hat{w}}}\left(D(I)_{w}\right)=\operatorname{gin}_{\sigma_{\hat{w}}}(I)=I
\end{aligned}
$$

Instead, if we do the same computation with $\sigma=$ DegRevLex, we get that both $\operatorname{gin}_{\sigma_{\hat{w}}}\left(D(I)_{h}\right)$ and $\operatorname{gin}_{\sigma_{\hat{w}}}\left(D(I)_{w}\right)$ coincide with $I$. We will see later that this fact is not a coincidence.

WARNING: As we said in the introduction, CoCoA examples involving computations of generic initial ideals, have probability of being correct equal to $100 \%-\varepsilon$, where $\varepsilon$ is as small as you wish... but not equal 0 . 


\section{Distractions}

We are looking for special situations where an equality of the type described in Theorem 1.13 holds, but with $h$ replaced by $x_{n}$. To achieve that goal, we need more preparatory results. In particular we need to build up some material around the notion of distraction. To this end, we split the entire section into three subsections, each addressing a specific question.

\section{A First Properties}

We start out with a definition which involves an infinite matrix of linear forms. The definition is given in that way in order to simplify the discussion. It will be clear, very soon, that in any application only a finite number of columns play a role. As before, $K$ is an infinite field, and $P=K\left[x_{1}, \ldots, x_{n}\right]$.

Definition 2.1. We let $\mathcal{L}=\left(L_{i j} \mid i=1, \ldots, n, j \in \mathbb{N}\right)$ be an infinite matrix with entries $L_{i j} \in P_{1}$ with the following two properties

a) The equality $\left\langle L_{1 j_{1}}, \ldots, L_{n j_{n}}\right\rangle=P_{1}$ holds for every $j_{1}, \ldots, j_{n} \in \mathbb{N}$.

b) There exist an integer $N \in \mathbb{N}$ such that $L_{i j}=L_{i N}$ for every $j>N$. We call $\mathcal{L}$ an $N$-distraction matrix or simply a distraction matrix.

Definition 2.2. Let $\mathcal{L}$ be a distraction matrix, and $t=x_{1}^{\alpha_{1}} x_{2}^{\alpha_{2}} \cdots x_{n}^{\alpha_{n}}$ a power product in $\mathbb{T}^{n}$. Then the polynomial $D_{\mathcal{L}}(t)=\prod_{i=1}^{n}\left(\prod_{j=1}^{\alpha_{j}} L_{i j}\right)$ is called the $\mathcal{L}$-distraction of $t$. Having defined $D_{\mathcal{L}}(t)$ for every power product $t$, we may consider $D_{\mathcal{L}}$ as an operator acting on the power products which can then be extended by linearity. Therefore we can write $D_{\mathcal{L}}(V)$ where $V$ is a subvector space of $P$, and call it the $\mathcal{L}$-distraction of $V$.

EXAMPLE 2.3. (Identical Distractions)

An obvious example of a distraction matrix is obtained by taking $L_{i j}=x_{i}$ for every $i, j$. In this case the operator $D_{\mathcal{L}}$ is the identity operator.

ExAmPle 2.4. (Classic Distractions)

A classic example of distraction matrix is given by choosing a big number $N$, and then considering the assignment $L_{i j}=x_{i}-(j-1) x_{n}$ for $i=1, \ldots, n-1$ and every $j<N, L_{n j}=x_{n}$ for every $j \in \mathbb{N}$, and $L_{i j}=x_{i}$ for every $j \geq N$. An important property of this distraction is that $D_{\mathcal{L}}(t)$ is square-free for every power product $t \in \mathbb{T}^{n-1}$, provided that $N$ and the characteristic of $K$ are greater than the maximum exponent in $t$.

EXAMPLE 2.5. (1-Distractions)

It is interesting to observe that a 1-distraction is nothing else than a linear change of coordinates. Therefore, if the linear forms $L_{11}, \ldots, L_{n 1}$ are generic, when we apply the 1-distraction to a vector space we get a generic linear change of coordinates. 


\section{EXAMPle 2.6. (Generic Distractions)}

We observe that an infinite matrix with entries $L_{i j} \in P_{1}$ and property b) is a distraction matrix if for every choice $j_{1}, \ldots, j_{n} \in \mathbb{N}$, the square matrix which represents $\left(L_{1 j_{1}}, \ldots, L_{n j_{n}}\right)$ with respect to the canonical basis $\left(x_{1}, \ldots, x_{n}\right)$ is invertible. We also observe that, due to property $b$ ), the number of matrices required to be invertible is finite. Therefore, if the matrix $\mathcal{L}$ has property $b$ ), and all its entries are generic up to column $N$, then $\mathcal{L}$ is an $N$-distraction matrix.

Later on, we shall need this notion of genericity.

Definition 2.7. A distraction matrix $\mathcal{L}$ is said to be sufficiently generic if $\left\langle L_{1 j_{1}}, \ldots, L_{k j_{k}}, x_{k+1}, \ldots, x_{n}\right\rangle=P_{1}$ for every $k=1, \ldots, n$.

A distraction matrix is sufficiently generic if for every choice $j_{1}, \ldots, j_{n} \in \mathbb{N}$, all the principal minors of the square matrix which represents $\left(L_{1 j_{1}}, \ldots, L_{n j_{n}}\right)$ with respect to the canonical basis $\left(x_{1}, \ldots, x_{n}\right)$, are invertible. Again, due to property b) of Definition 2.1, the number of minors required to be invertible is finite.

We observe that for every power product $t$, we have $\operatorname{deg}(t)=\operatorname{deg}\left(D_{\mathcal{L}}(t)\right)$, therefore, we can view $D_{\mathcal{L}}$ as a $K$-linear operator on every $P_{d}$. Moreover, if $t_{1}, t_{2}$ are power products such that $t_{1} \mid t_{2}$, then $D_{\mathcal{L}}\left(t_{1}\right) \mid D_{\mathcal{L}}\left(t_{2}\right)$, but of course it is not true that $D_{\mathcal{L}}\left(t_{1} t_{2}\right)=D_{\mathcal{L}}\left(t_{1}\right) D_{\mathcal{L}}\left(t_{2}\right)$, so $D_{\mathcal{L}}: P \longrightarrow P$ is not a homomorphism of rings.

Proposition 2.8 Let $\mathcal{L}$ be a distraction matrix, and let $\mathfrak{g} \in \mathrm{GL}(n, K)$ be a matrix.

a) The matrix $\mathfrak{g} \cdot \mathcal{L}=\left(\mathfrak{g} \cdot \mathcal{L}_{i j}\right)$ is a distraction matrix.

b) We have $\mathfrak{g} D_{\mathcal{L}}=D_{\mathfrak{g} \cdot \mathcal{L}}$.

c) If $\mathfrak{g}$ is generic, then $\mathfrak{g} \cdot \mathcal{L}$ is sufficiently generic.

Proof. Since a) is clear, we move to the proof of b). It is sufficient to prove the equality $\mathfrak{g}\left(D_{\mathcal{L}}(t)\right)=D_{\mathfrak{g} \cdot \mathcal{L}}(t)$ for every power product $t$. And such equality stems directly from the definitions.

Let us prove c). We have already observed right after the definition of a sufficiently generic matrix that the number of minors required to be invertible is finite. Let $\mathfrak{g}$ be a matrix whose entries are distinct indeterminates, and let $\mathfrak{m}_{1}, \ldots, \mathfrak{m}_{s}$ be the square invertible matrices which represent the $n$-tuples $\left(L_{1 j_{1}}, \ldots, L_{n j_{n}}\right)$ with respect to the canonical basis $\left(x_{1}, \ldots, x_{n}\right)$, and which are required to have invertible principal minors. Now every matrix $\mathfrak{m}_{i}$ can be put in triangular form with a suitable change of coordinates. Therefore the condition on $\mathfrak{g}$ to transform $\mathfrak{m}_{i}$ into a matrix with the desired property is open and non-empty. Since we have to achieve the property on a finite number of matrices, we see that the condition on $\mathfrak{g}$ is open and non-empty. Now the proof is complete. 
The following proposition and subsequent corollary allow us to extend the notion of $\mathcal{L}$-distractions to ideals. If $f$ is a homogeneous polynomial $d$, we use the notation $f P_{1}$ to indicate the subvector space $\left\langle f x_{1}, \ldots, f x_{n}\right\rangle$ of $P_{d+1}$. More generally, if $V$ is a subvector space of $P_{d}$ we use the notation $V P_{1}$ to indicate the subvector space of $P_{d+1}$ spanned by the set $\left\{v x_{i} \mid v \in V, i=1, \ldots, n\right\}$.

Proposition 2.9 Let $\mathcal{L}$ be a distraction matrix.

a) We have $D_{\mathcal{L}}\left(V P_{d}\right)=D_{\mathcal{L}}(V) P_{d}$ for every monomial vector space $V$, and every $d \in \mathbb{N}$.

b) The map $D_{\mathcal{L}}$ induces a $K$-linear automorphism of $P_{d}$ for every $d \in \mathbb{N}$.

c) We have $\operatorname{dim}_{K}(V)=\operatorname{dim}_{K}\left(D_{\mathcal{L}}(V)\right)$ for every subvector space $V$ of $P$.

d) Let $V, W$ be subvector spaces of $P$. Then $D_{\mathcal{L}}(V \cap W)=D_{\mathcal{L}}(V) \cap D_{\mathcal{L}}(W)$.

e) Let $V, W$ be subvector spaces of $P$. Then $D_{\mathcal{L}}(V+W)=D_{\mathcal{L}}(V)+D_{\mathcal{L}}(W)$.

Proof. We let $t=x_{1}^{\alpha_{1}} x_{2}^{\alpha_{2}} \cdots x_{n}^{\alpha_{n}}$ be a power product in $\mathbb{T}^{n}$. Then $D_{\mathcal{L}}\left(t P_{1}\right)=$ $\left\langle D_{\mathcal{L}}\left(t x_{1}\right), \ldots, D_{\mathcal{L}}\left(t x_{n}\right)\right\rangle=\left\langle D_{\mathcal{L}}\left(x_{1}^{\alpha_{1}+1} x_{2}^{\alpha_{2}} \cdots x_{n}^{\alpha_{n}}\right), \ldots, D_{\mathcal{L}}\left(x_{1}^{\alpha_{1}} x_{2}^{\alpha_{2}} \cdots x_{n}^{\alpha_{n}+1}\right)\right\rangle=$ $\left\langle D_{\mathcal{L}}(t) L_{1, \alpha_{1}+1}, \ldots, D_{\mathcal{L}}(t) L_{n, \alpha_{n}+1}\right\rangle=D_{\mathcal{L}}(t)\left\langle L_{1, \alpha_{1}+1}, \ldots, L_{n, \alpha_{n}+1}\right\rangle=D_{\mathcal{L}}(t) P_{1}$, where the last equality follows from the assumption that $\mathcal{L}$ is a distraction matrix. We have proved that $D_{\mathcal{L}}\left(t P_{1}\right)=D_{\mathcal{L}}(t) P_{1}$ which implies the equality $D_{\mathcal{L}}\left(V P_{1}\right)=D_{\mathcal{L}}(V) P_{1}$. Now we prove the claim by induction, namely we assume that $D_{\mathcal{L}}\left(V P_{d-1}\right)=D_{\mathcal{L}}(V) P_{d-1}$, and get

$$
D_{\mathcal{L}}\left(V P_{d}\right)=D_{\mathcal{L}}\left(V P_{d-1} P_{1}\right)=D_{\mathcal{L}}\left(V P_{d-1}\right) P_{1}=D_{\mathcal{L}}(V) P_{d-1} P_{1}=D_{\mathcal{L}}(V) P_{d}
$$

The proof of a) is now complete.

To prove b) we observe that, applying a) to $V=K$, we get $D_{\mathcal{L}}\left(P_{d}\right)=$ $P_{d}$, hence the $K$-linear operator $D_{\mathcal{L}}: P_{d} \longrightarrow P_{d}$ is surjective and hence an automorphism.

The proofs of c), d) and e) follow directly from b).

In the following, if $M$ is a standard graded $P$-module, we use the notation $\mathrm{HF}_{M}$ to indicate its Hilbert function.

\section{Corollary 2.10 (Distractions and Hilbert Functions)}

Let $\mathcal{L}$ be a distraction matrix, and $I$ a monomial ideal in $P$.

a) The vector space $D_{\mathcal{L}}(I)$ coincides with $\oplus_{d} D_{\mathcal{L}}\left(I_{d}\right)$, and is a homogeneous ideal in $P$.

b) Let $t_{1}, \ldots, t_{r} \in \mathbb{T}^{n}$ be such that $I=\left(t_{1}, \ldots, t_{r}\right)$. Then we have the equality $D_{\mathcal{L}}(I)=\left(D_{\mathcal{L}}\left(t_{1}\right), \ldots, D_{\mathcal{L}}\left(t_{r}\right)\right)$.

c) We have $\mathrm{HF}_{I}=\operatorname{HF}_{D_{\mathcal{L}}(I)}$.

Proof. The fact that $D_{\mathcal{L}}(I)=\oplus_{d} D_{\mathcal{L}}\left(I_{d}\right)$ follows from the definition. To prove that it is a homogeneous ideal, we have to show that $D_{\mathcal{L}}\left(I_{d}\right) P_{d^{\prime}} \subseteq D_{\mathcal{L}}\left(I_{d+d^{\prime}}\right)$ for every $d, d^{\prime} \in \mathbb{N}$. Indeed, $D_{\mathcal{L}}\left(I_{d}\right) P_{d^{\prime}}=D_{\mathcal{L}}\left(I_{d} P_{d^{\prime}}\right)$ by Proposition 2.9.a, and the conclusion follows. To prove b), we show that $D_{\mathcal{L}}(I)_{d}=\left(D_{\mathcal{L}}\left(t_{1}\right), \ldots, D_{\mathcal{L}}\left(t_{r}\right)\right)_{d}$ 
for every $d \in \mathbb{N}$. To this end, we observe that $I_{d}=t_{1} P_{d-d_{1}}+\cdots+t_{r} P_{d-d_{r}}$, where $d_{j}=\operatorname{deg}\left(t_{j}\right)$ for $j=1, \ldots, r$. Therefore

$D_{\mathcal{L}}\left(I_{d}\right)=D_{\mathcal{L}}\left(t_{1} P_{d-d_{1}}\right)+\cdots D_{\mathcal{L}}\left(t_{r} P_{d-d_{r}}\right)=D_{\mathcal{L}}\left(t_{1}\right) P_{d-d_{1}}+\cdots+D_{\mathcal{L}}\left(t_{r}\right) P_{d-d_{r}}$

where the second equality follows from Proposition 2.9.a, and the proof is complete. Finally, claim c) is an immediate consequence of Proposition 2.9.c.

The next result relates the operations of saturating and distracting monomial ideals. We use the notation $\operatorname{Sat}(I)$ to indicate the saturation of $I$, i.e. the unique ideal $J$ such that $\operatorname{depth}(P / J)>0$ and $J_{i}=I_{i}$ for $i>>0$.

Corollary 2.11 (Distractions and Saturation)

Let $\mathcal{L}$ be a distraction matrix and let $I$ be a monomial ideal in $P$. Then $D_{\mathcal{L}}(\operatorname{Sat}(I))=\operatorname{Sat}\left(D_{\mathcal{L}}(I)\right)$.

Proof. We denote by $M$ the ideal $\left(x_{1}, \ldots, x_{n}\right)$ and observe that $t \in I: M^{r}$ if and only if $t P_{r} \subseteq I$ which is equivalent to $D_{\mathcal{L}}\left(t P_{r}\right) \subseteq D_{\mathcal{L}}(I)$ by Proposition 2.9.b. Now $D_{\mathcal{L}}\left(t P_{r}\right) \subseteq D_{\mathcal{L}}(I)$ is equivalent to $D_{\mathcal{L}}(t) P_{r} \subseteq D_{\mathcal{L}}(I)$ by Proposition 2.9.a. which is equivalent to $D_{\mathcal{L}}(t) \in D_{\mathcal{L}}(I): M^{r}$, and the proof is complete.

\section{B Distractions and Betti numbers}

We have just proved that $I$ and $D_{\mathcal{L}}(I)$ share the same Hilbert function. Now we are going to prove a stronger property, namely that $I$ and $D_{\mathcal{L}}(I)$ share also the same Betti numbers.

To achieve that result we need more facts about multigraded rings and modules. A full treatment of this subject can be found in [KR04], from which we borrow some basic statements.

Definition 2.12. Let $m \geq 1$, and let the polynomial ring $P=K\left[x_{1}, \ldots, x_{n}\right]$ be equipped with a $\mathbb{Z}^{m}$-grading such that $K \subseteq P_{0}$ and $x_{1}, \ldots, x_{n}$ are homogeneous elements.

a) For $j=1, \ldots, n$, let $\left(w_{1 j}, \ldots, w_{m j}\right) \in \mathbb{Z}^{m}$ be the degree of $x_{j}$. The matrix $W=\left(w_{i j}\right) \in \operatorname{Mat}_{m, n}(\mathbb{Z})$ is called the degree matrix of the given grading. The rows of this matrix are called the weight vectors of the indeterminates $x_{1}, \ldots, x_{n}$.

b) Conversely, given a matrix $W=\left(w_{i j}\right) \in \operatorname{Mat}_{m, n}(\mathbb{Z})$, we can consider the $\mathbb{Z}^{m}$-grading on $P$ such that $K \subseteq P_{0}$ and such that the indeterminates are homogeneous elements whose degrees are given by the columns of $W$. In this case, we say that $P$ is multigraded by $W$, or simply graded by $W$.

c) Let $d \in \mathbb{Z}^{m}$. The set of homogeneous polynomials of degree $d$ is denoted by $P_{W, d}$, or simply by $P_{d}$ if it is clear which grading we are considering. A polynomial $f \in P_{W, d}$ is also called homogeneous of multidegree $d$, or simply of degree $d$, and we write $\operatorname{deg}_{W}(f)=d$. 
Proposition 2.13 Let $I$ be an ideal of $P$. Then the following conditions are equivalent.

a) The ideal $I$ is a monomial ideal.

b) There is a non-singular matrix $W \in \operatorname{Mat}_{n}(\mathbb{Z})$ such that $I$ is a homogeneous ideal with respect to the grading on $P$ given by $W$.

c) For every $m \geq 1$ and every matrix $W \in \operatorname{Mat}_{m, n}(\mathbb{Z})$, the ideal $I$ is a homogeneous ideal with respect to the grading on $P$ given by $W$.

Proof. The easy proof is left to the reader.

This result allows us to say that a monomial ideal $J$ is $\mathbb{I}$-homogeneous, where $\mathbb{I}$ is the identity matrix. Therefore, we may construct a minimal finite free resolution of $J$, which is also graded by $\mathbb{I}$. To understand it, it is therefore important to study $\mathbb{I}$-graded modules and homomorphisms.

Clearly, the $\mathbb{I}$-degree of a power product $t$ in $P$ is $\log (t)$, so that we can identify $t$ with its $\mathbb{I}$-degree, and write $\operatorname{deg}_{\mathbb{I}}(t)=t$. Consequently, the graded free $P$-module generated by 1 with $\operatorname{deg}_{\mathbb{I}}(1)=t$, can be written as $P(-t)$, instead of $P(-\log (t))$.

Next, we characterize $\mathbb{I}$-graded free modules, their $\mathbb{I}$-graded submodules, and $\mathbb{I}$-graded homomorphisms.

\section{Proposition 2.14 (I-Graded Free Modules and Homomorphisms)}

Let $P$ be graded by $\mathbb{I}$.

a) Let $\oplus_{i=1}^{r} P\left(-t_{i}\right)$ be a graded free $P$-module, let $t \in \mathbb{T}^{n}$, and let $v$ be a homogeneous vector of degree $t$ in $\oplus_{i=1}^{r} P\left(-t_{i}\right)$. Then $v=\left(c_{1} s_{1}, \ldots, c_{r} s_{r}\right)$ where $c_{i} \in K, s_{i} \in \mathbb{T}^{n}$, and $s_{i} t_{i}=t$ for every $i=1, \ldots, r$.

b) Let $\oplus_{i=1}^{r} P\left(-t_{i}\right), \oplus_{i=1}^{r^{\prime}} P\left(-t_{i}^{\prime}\right)$ be finitely generated graded free $P$-modules, and $\oplus_{i=1}^{r} P\left(-t_{i}\right) \stackrel{\varphi}{\longrightarrow} \oplus_{i=1}^{r^{\prime}} P\left(-t_{i}^{\prime}\right)$ a graded $P$-homomorphism. If $\varphi$ is represented by a matrix $\mathcal{M}=\left(m_{i j}\right)$ with respect to the canonical bases, then we have $m_{i j}=0$ if $t_{i}^{\prime}$ does not divide $t_{j}$, and $m_{i j}=c_{i j} t_{j} / t_{i}^{\prime}$ with $c_{i j} \in K$, if $t_{i}^{\prime}$ divides $t_{j}$.

Proof. The proof of a) follows from the remark that monomials are the only homogeneous elements in $P$. To prove b), we observe that $m_{i j}$ is the $i^{\text {th }}$ coordinate of $\varphi\left(e_{j}\right)$. Now, $\operatorname{deg}\left(e_{j}\right)=t_{j}$, hence $\operatorname{deg}\left(\varphi\left(e_{j}\right)\right)=t_{j}$. But the only elements of degree $t_{j}$ in $P\left(-t_{i}^{\prime}\right)$ are the monomials $c_{i j} t_{j} / t_{i}^{\prime}$ if $t_{i}^{\prime}$ divides $t_{j}$. Instead, if $t_{i}^{\prime}$ does not divide $t_{j}$, only 0 is homogeneous of degree $t_{j}$ in $P\left(-t_{i}^{\prime}\right)$.

\section{Corollary 2.15 (I-Graded Free Submodules)}

Let $P$ be graded by $\mathbb{I}$, let $\oplus_{i=1}^{r} P\left(-t_{i}\right)$ be an $\mathbb{I}$-graded free module, and let $M \subseteq \oplus_{i=1}^{r} P\left(-t_{i}\right)$ be an $\mathbb{I}$-graded submodule. Then $M$ is generated by a finite set of homogeneous vectors, i.e. vectors of the type $v=\left(c_{1} s_{1}, \ldots, c_{r} s_{r}\right)$ where $c_{i} \in K, s_{i} \in \mathbb{T}^{n}$, and $s_{1} t_{1}=s_{2} t_{2}=\cdots=s_{r} t_{r}$. 
Let us look at Proposition 2.14.c. For the sake of simplicity, we are going to use the convention that if $g$ divides $f$, and hence $f=g h$, the symbol $f:: g$ means $h$, otherwise it means 0 . After the description given in the above proposition, we allow ourselves to write $\mathcal{M}=\left(m_{i j}\right)=\left(c_{i j} t_{j}:: t_{i}^{\prime}\right)$.

Let us turn to distractions. We have seen that the operator $D_{\mathcal{L}}$ acts on power products and extends to a $K$-linear map $D_{\mathcal{L}}: P \longrightarrow P$ which is not a homomorphism of rings. To see how it extends to $\mathbb{I}$-graded free $P$-modules, let $\oplus_{i=1}^{r} P\left(-t_{i}\right)$ be an $\mathbb{I}$-graded free $P$-module, let $d_{i}$ be the standard degree of $t_{i}$, and consider the standard graded free $P$-module $\oplus_{i=1}^{r} P\left(-d_{i}\right)$.

We have already observed that $t_{1} \mid t_{2}$ implies $D_{\mathcal{L}}\left(t_{1}\right) \mid D_{\mathcal{L}}\left(t_{2}\right)$, and this fact allows us to give the following definition.

Definition 2.16. We define a map from $\oplus_{i=1}^{r} P\left(-t_{i}\right)$ to $\oplus_{i=1}^{r} P\left(-d_{i}\right)$ by sending every power product $t e_{i}$ to $D_{\mathcal{L}}\left(t t_{i}\right) / D_{\mathcal{L}}\left(t_{i}\right) e_{i}$. Then we extend it uniquely to a $K$-linear map which preserves the standard degrees, and which we call $D_{\mathcal{L}}$.

REMARK 2.17. Arguing as in Corollary 2.10 and its proof, we see that if $M$ is an $\mathbb{I}$-graded submodule of $\oplus_{i=1}^{r} P\left(-t_{i}\right)$ (as described in Corollary 2.15), then $D_{\mathcal{L}}(M)$ is a standard graded submodule of $\oplus_{i=1}^{r} P\left(-d_{i}\right)$. Moreover, they share the same Hilbert function.

Next, we describe how the homomorphisms of $\mathbb{I}$-graded free modules, and the maps $D_{\mathcal{L}}$ work together.

Lemma 2.18 Let $\oplus_{i=1}^{r} P\left(-t_{i}\right)$ and $\oplus_{i=1}^{r^{\prime}} P\left(-t_{i}^{\prime}\right)$ be $\mathbb{I}$-graded free $P$-modules, let $d_{i}=\operatorname{deg}\left(t_{i}\right), d_{i}^{\prime}=\operatorname{deg}\left(t_{j}\right)$ where $\operatorname{deg}$ is the standard degree, and let $\varphi: \oplus_{i=1}^{r} P\left(-t_{i}\right) \longrightarrow \oplus_{i=1}^{r^{\prime}} P\left(-t_{i}^{\prime}\right)$ be a homomorphism of $\mathbb{I}$-graded $P$-modules. Assume that $\varphi$ is represented by the matrix $\mathcal{M}=\left(m_{i j}\right)=\left(c_{i j} t_{j}:: t_{i}^{\prime}\right)$, let $D_{\mathcal{L}}(\mathcal{M})=\left(c_{i j} D_{\mathcal{L}}\left(t_{j}\right):: D_{\mathcal{L}}\left(t_{i}^{\prime}\right)\right)$, and let $D_{\mathcal{L}}(\varphi): \oplus_{i=1}^{r} P\left(-d_{i}\right) \longrightarrow \oplus_{i=1}^{r^{\prime}} P\left(-d_{i}^{\prime}\right)$ be the standard graded homomorphism of $P$-modules defined by $D_{\mathcal{L}}(\mathcal{M})$.

a) The maps $D_{\mathcal{L}}$ are isomorphisms of $K$ vector spaces which preserve the standard degree.

b) The maps $\varphi$ and $D_{\mathcal{L}}(\varphi)$ preserve the standard degrees.

c) The diagram

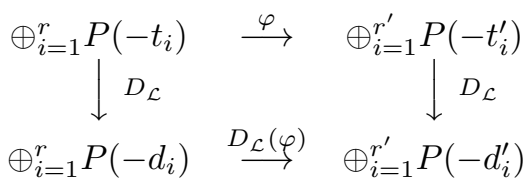

is a commutative diagram of $K$-vector spaces.

Proof. We have already observed that $D_{\mathcal{L}}$ preserves the standard degrees. It is an isomorphism by Proposition 2.9.b. So the proof of a) is complete. 
We prove b). The map $\varphi$ is a homomorphism of $\mathbb{I}$-graded $P$-modules, therefore it preserves the fine degrees, and hence the standard degrees too. Finally, we have $\operatorname{deg}\left(D_{\mathcal{L}}\left(t_{j}\right) \mid D_{\mathcal{L}}\left(t_{i}^{\prime}\right)\right)=d_{j}-d_{i}^{\prime}$, which implies that $D_{\mathcal{L}}(\varphi)$ preserves the standard degrees.

To show the commutativity of the diagram, it suffices to pick an element of type $t e_{j} \in \oplus_{i=1}^{r} P\left(-t_{i}\right)$ and show that $D_{\mathcal{L}}\left(\varphi\left(t e_{j}\right)\right)=D_{\mathcal{L}}(\varphi)\left(D_{\mathcal{L}}\left(t e_{j}\right)\right)$, and to prove such equality it suffices to prove it componentwise. Let us compute the $i$-component of both vectors. The $i$-component of $D_{\mathcal{L}}\left(\varphi\left(t e_{j}\right)\right)$ is

$$
D_{\mathcal{L}}\left(c_{i j} t\left(t_{j}:: t_{i}^{\prime}\right)\right)=c_{i j} D_{\mathcal{L}}\left(t_{i}^{\prime} t\left(t_{j}:: t_{i}^{\prime}\right)\right) / D_{\mathcal{L}}\left(t_{i}^{\prime}\right)
$$

and the latter is either $c_{i j} D_{\mathcal{L}}\left(t t_{j}\right) / D_{\mathcal{L}}\left(t_{i}^{\prime}\right)$ or 0 . The $i$-component of the vector $D_{\mathcal{L}}(\varphi)\left(D_{\mathcal{L}}\left(t e_{j}\right)\right)$ is the $i$-component of $D_{\mathcal{L}}(\varphi)\left(c_{i j} D_{\mathcal{L}}\left(t t_{j}\right) / D_{\mathcal{L}}\left(t_{j}\right) e_{j}\right)$, ie.

$$
c_{i j} D_{\mathcal{L}}\left(t t_{j}\right) / D_{\mathcal{L}}\left(t_{j}\right)\left(D_{\mathcal{L}}\left(t_{j}\right):: D_{\mathcal{L}}\left(t_{i}^{\prime}\right)\right)
$$

and the latter is either $c_{i j} D_{\mathcal{L}}\left(t t_{j}\right) / D_{\mathcal{L}}\left(t_{i}^{\prime}\right)$ or 0 . The proof is complete.

Using the above terminology, we are ready to prove an interesting result.

\section{Theorem 2.19 (Distractions and Homology)}

Let $\oplus_{i=1}^{r^{\prime \prime}} P\left(-t_{i}^{\prime \prime}\right) \stackrel{\psi}{\longrightarrow} \oplus_{i=1}^{r} P\left(-t_{i}\right) \stackrel{\varphi}{\longrightarrow} \oplus_{i=1}^{r^{\prime}} P\left(-t_{i}^{\prime}\right)$ be a complex of $\mathbb{I}$-graded free modules and homomorphisms. Let $D_{\mathcal{L}}, D_{\mathcal{L}}(\psi), D_{\mathcal{L}}(\varphi)$ be defined as above.

a) The diagram

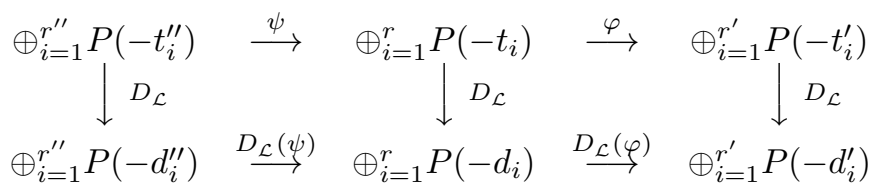

is commutative.

b) We have $D_{\mathcal{L}}(\operatorname{Ker}(\varphi))=\operatorname{Ker}\left(D_{\mathcal{L}}(\varphi)\right)$, and $D_{\mathcal{L}}(\operatorname{Im}(\varphi))=\operatorname{Im}\left(D_{\mathcal{L}}(\varphi)\right)$.

c) The map $D_{\mathcal{L}}: \oplus_{i=1}^{r} P\left(-t_{i}\right) \longrightarrow \oplus_{i=1}^{r} P\left(-d_{i}\right)$ induces a $K$-linear map

$$
\bar{D}_{\mathcal{L}}: \operatorname{Ker}(\varphi) / \operatorname{Im}(\psi) \longrightarrow \operatorname{Ker}\left(D_{\mathcal{L}}(\varphi)\right) / \operatorname{Im}\left(D_{\mathcal{L}}(\psi)\right)
$$

which is compatible with the standard degree.

d) The map $\bar{D}_{\mathcal{L}}$ is an isomorphism of vector spaces.

Proof. Claim a) follows from Lemma 2.18.c. The horizontal maps preserve the standard degrees by Lemma 2.18.b., and the vertical maps are isomorphisms of vector spaces which preserve the standard degree by Lemma 2.18.a. The other conclusions are achieved with standard arguments in homological algebra.

Finally we can see the relationship between the Betti numbers of $\mathbb{I}$-graded modules and their distractions. 


\section{Corollary 2.20 (Distractions and Betti Numbers)}

Let $\mathcal{L}$ be a distraction matrix, and $M$ an $\mathbb{I}$-graded submodule of a finitely generated $\mathbb{I}$-graded free module. The standard Betti numbers of $M$ and $D_{\mathcal{L}}(M)$ are the same.

Proof. Let be given a minimal finite free $\mathbb{I}$-graded resolution of $M$. We apply the operator $D_{\mathcal{L}}$ to the entire resolution, and the theorem shows that we get a minimal finite free standard graded resolution of $D_{\mathcal{L}}(M)$. Since the maps $D_{\mathcal{L}}$ are isomorphisms of $K$-vector spaces, the standard Betti numbers of $M$ and $D_{\mathcal{L}}(M)$ are indeed the same.

\section{C Radical Distractions}

The final part of this section addresses the following problem. If $I$ is a monomial ideal, under which conditions on $\mathcal{L}$ is $D_{\mathcal{L}}(I)$ a radical ideal? We recall that irreducible monomial ideals are of the type $\left(x_{i_{1}}^{a_{1}}, \ldots, x_{i_{h}}^{a_{h}}\right)$, and that every monomial ideal is the intersection of irreducible monomial ideals, called irreducible components.

Definition 2.21. Let $I=\left(x_{i_{1}}^{a_{1}}, \ldots, x_{i_{h}}^{a_{h}}\right)$ be an irreducible monomial ideal in $P$, and let $S=\left\{\left(s_{1}, \ldots, s_{h}\right) \mid 1 \leq s_{1} \leq a_{1}, \ldots, 1 \leq s_{h} \leq a_{h}\right\}$. Then let $\mathcal{L}$ be a distraction matrix, and let $V_{\left(s_{1}, \ldots, s_{h}\right)}$ be the $K$-vector space generated by $\left\{L_{i_{1} s_{1}}, \ldots, L_{i_{h} s_{h}}\right\}$. If the vector spaces $V_{\left(s_{1}, \ldots, s_{h}\right)}$ are pairwise distinct when $\left(s_{1}, \ldots, s_{h}\right)$ spans $S$, we say that $\mathcal{L}$ is radical for $I$. We observe that a necessary condition for an $N$-distraction matrix to be radical for $I$ is that $N \geq a_{r}$ for $r=1, \ldots, h$.

More generally, if $I$ is any monomial ideal, we say that $\mathcal{L}$ is radical for $I$ if $\mathcal{L}$ is radical for all the irreducible components of $I$.

We observe that, with the exception of the case $I=\left(x_{1}, \ldots, x_{n}\right)$, a necessary condition for a distraction matrix to be radical for $I$ is that $\operatorname{depth}(P / I)>0$, since the vector spaces spanned by the linear forms taken one from each of the rows coincide with $P_{1}$.

EXAMPLE 2.22. The identical distractions of Example 2.3 are clearly not radical for any irreducible monomial ideal, except the linear ones. Instead, the $N$-generic distractions of Example 2.5 are radical for every monomial ideal $I$ such that $\operatorname{depth}(P / I)>0$, only provided that $N$ is sufficiently big.

EXAMPLE 2.23. Let us consider the monomial ideal $I=\left(x_{1}^{2} x_{2}^{2}, x_{1}^{2} x_{3}^{2}, x_{2}^{2} x_{3}^{2}\right)$ in $P=K\left[x_{1}, x_{2}, x_{3}\right]$. Then

$$
I=\left(x_{1}^{2}, x_{2}^{2}\right) \cap\left(x_{1}^{2}, x_{3}^{2}\right) \cap\left(x_{2}^{2}, x_{3}^{2}\right)
$$

is the decomposition of $I$ into irreducible components. Now it is clear that the generic 2-distraction is radical for $I$, while the classic 2 -distraction is not. 
Next result provides a reason for the word radical used in the definition above.

Lemma 2.24 Let $h<n$, let $I=\left(x_{i_{1}}^{a_{1}}, \ldots, x_{i_{h}}^{a_{h}}\right)$ be an irreducible monomial ideal, let $S=\left\{\left(s_{1}, \ldots, s_{h}\right) \mid 1 \leq s_{1} \leq a_{1}, \ldots, 1 \leq s_{h} \leq a_{h}\right\}$, and let $\mathcal{L}$ be a distraction matrix which is radical for $I$.

a) We have $D_{\mathcal{L}}(I)=\bigcap_{\left(s_{1}, \ldots, s_{h}\right) \in S}\left(L_{i_{1} s_{1}}, \ldots, L_{i_{h} s_{h}}\right)$.

b) The ideal $D_{\mathcal{L}}(I)$ is radical.

Proof. Clearly b) follows immediately from a), and we prove a) by induction on $h$. The assumption on $\mathcal{L}$ implies that $L_{i_{r} 1}, L_{i_{r} 2}, \ldots, L_{i_{r} a_{r}}$ are pairwise coprime for every $r=1, \ldots, h$. Therefore if $h=1$ the claim follows. Let $h>1$ and write $I=\left(x_{i_{1}}^{a_{1}}\right)+J$, where $J=\left(x_{2}^{a_{2}}, \ldots, x_{h}^{a_{h}}\right)$. Then $D_{\mathcal{L}}(I)=$ $\left(D_{\mathcal{L}}\left(x_{1}^{a_{1}}\right)\right)+D_{\mathcal{L}}(J)$. By induction, we have $D_{\mathcal{L}}(J)=\bigcap_{\left(s_{2}, \ldots, s_{h}\right)}\left(L_{2 s_{2}}, \ldots, L_{h s_{h}}\right)$. Each ideal showing up in the intersection is a linear, and hence prime, ideal of height $h-1$. Now, $L_{i_{1} 1}, \ldots, L_{i_{1} a_{1}}$ are pairwise coprime and they do not divide zero modulo any of the prime ideals in the intersection. Standard facts in ideal theory imply that $D_{\mathcal{L}}(J)=\bigcap_{\left(s_{1}, \ldots, s_{h}\right)}\left(L_{1 s_{1}}, \ldots, L_{h s_{h}}\right)$, and the proof is complete.

REMARK 2.25. An alternative way of proving the above lemma goes as follows. Since $\left(x_{i_{1}}^{a_{1}}, \ldots, x_{i_{h}}^{a_{h}}\right)$ is a regular sequence, a direct argument or an appropriate use of Theorem 2.19, shows that $\left(D_{\mathcal{L}}\left(x_{i_{1}}^{a_{1}}\right), \ldots, D_{\mathcal{L}}\left(x_{i_{h}}^{a_{h}}\right)\right)$ is a regular sequence too. This fact implies that $D_{\mathcal{L}}(I)$ is pure. Now all the ideals showing up in the intersection are linear, hence prime of multiplicity 1. Clearly $D_{\mathcal{L}}(I) \subset \bigcap_{\left(s_{1}, \ldots, s_{h}\right)}\left(L_{1 s_{1}}, \ldots, L_{h s_{h}}\right)$, and both ideals are pure and have the same dimension. From the assumption we deduce that all the ideals in the intersection are pairwise distinct, hence $D_{\mathcal{L}}(I)$ and $\bigcap_{\left(s_{1}, \ldots, s_{h}\right)}\left(L_{1 s_{1}}, \ldots, L_{h s_{h}}\right)$ have the same multiplicity too. So they are equal.

\section{Proposition 2.26 (Distractions and Radical Ideals)}

Let $I$ be a monomial ideal such that $\operatorname{depth}(P / I)>0$, and let $\mathcal{L}$ be a distraction matrix which is radical for $I$. Then the ideal $D_{\mathcal{L}}(I)$ is radical.

Proof. We express $I$ as the intersection of irreducible ideals. The assumption implies that every such irreducible ideal $J$ is such that $\operatorname{depth}(P / J)>0$. Therefore, after Proposition 2.9.d we may assume that $I$ is irreducible and that $\operatorname{depth}(P / I)>0$, hence that it is generated by $h<n$ pure power products. At this point it suffices to apply the lemma. 


\section{$3 \quad$ Stable and Strongly Stable Ideals}

In Section 1 we introduced stable and strongly stable ideals. Now we want to investigate their structure more closely.

Definition 3.1. Let $S=\left\{t_{1}, \ldots, t_{s}\right\}$ be a set of power products in $\mathbb{T}^{n}$. We denote by $\operatorname{Stable}(S)$ (SStable $(S))$ the smallest stable (strongly stable) ideal containing the elements of $S$. If $S=\{t\}$ then we simply write $\operatorname{Stable}(t)$ for $\operatorname{Stable}(S)$, and $\operatorname{SStable}(t)$ for $\operatorname{SStable}(S)$.

DEFINITION 3.2. If $t$ is a power product such that $x_{j} \mid t$ and $i \leq j$, we say that $x_{i} \frac{t}{x_{j}}$ is obtained from $t$ with an elementary move. Likewise, we say that $x_{i} \frac{t}{x_{\mathrm{m}(t)}}$ is obtained from $t$ with a special elementary move.

Then we say that $t^{\prime}$ is obtained from $t$ with a chain of (special) elementary moves, if there are power products $t_{1}, \ldots, t_{s}$ such that $t=t_{1}, t^{\prime}=t_{s}$, and $t_{i}$ is obtained from $t_{i-1}$ with a (special) elementary move for $i=2, \ldots, s$.

Using this terminology, we see that for a (stable) strongly stable set $T$, every power product reached from a power product in $T$ with a chain of (special) elementary moves, still lies in $T$.

It is clear that if $I=\operatorname{SStable}(S)(I=\operatorname{Stable}(S))$, every minimal generator of $I$ can be obtained by a chain of elementary moves (special elementary moves) from some elements in $S$. But we can say more.

Lemma 3.3 Let $r \leq n$, let $t_{1}, \ldots, t_{r} \in \mathbb{T}^{n}$, let $\alpha_{1}, \ldots, \alpha_{r}$ be non-negative integers, and let $I$ be the ideal $\sum_{j=1}^{r} t_{j}\left(x_{1}, \ldots, x_{j}\right)^{\alpha_{j}}$. Assume that $t_{1}=1$, $\mathrm{m}\left(t_{j}\right)<j$ for every $j=2, \ldots, r$, that $t_{j} \mid t_{j+1}$ for $1 \leq j<r$, and that $\operatorname{deg}\left(t_{j}\right)+$ $\alpha_{j} \leq \operatorname{deg}\left(t_{j+1}\right)+\alpha_{j+1}$ for all $1 \leq j<r$. Then

a) The ideal $I$ is stable.

b) The graded Betti numbers and hence Hilbert function of I only depend on the $r$-tuple of pairs $\left(\left(\operatorname{deg}\left(t_{1}\right), \alpha_{1}\right), \ldots,\left(\operatorname{deg}\left(t_{r}\right), \alpha_{r}\right)\right)$.

Proof. Let $I_{j}=t_{j}\left(x_{1}, \ldots, x_{j}\right)^{\alpha_{j}}$. We claim that $I$ is stable. For, let $t$ be a power product in $I$ and let $j$ be the smallest integer such that $t \in I_{j}$. Then $\mathrm{m}(t)=j$, otherwise by assumption we would have $t \in I_{j-1}$. We may write $t=t_{j} u$, where $u$ is a power product of degree $\alpha_{j}$ such that $\mathrm{m}(u)=j$. Then clearly $t x_{i} / x_{j}$ is in $I_{j}$ and hence in $I$ for all $i<j$.

A minimal free resolution of a stable monomial ideal $J$ is described by Eliahou and Kervaire [EK90]. It follows from their work that the graded Betti numbers of the stable ideal $J$ are determined by the multi-set of pairs of integers

$$
E K(J):=\{(\mathrm{m}(t), \operatorname{deg}(t)) \mid t \text { is a minimal generator of } J\}
$$

A minimal generator of $I$ is of the form $t_{j} u$ where $u$ is a power product of degree $\alpha_{j}, \mathrm{~m}(u)=j$, and the exponent of $x_{j}$ in $u$ is bigger than $\operatorname{deg}\left(t_{j}\right)+$ $\alpha_{j}-\operatorname{deg}\left(t_{j-1}\right)-\alpha_{j-1}$. So the multi-set $E K(I)$ only depends on the set of pairs $\left(\operatorname{deg}\left(t_{j}\right), \alpha_{j}\right)$ with $1 \leq j \leq r$. 
Proposition 3.4 Let $t=x_{1}^{a_{1}} \cdots x_{n}^{a_{n}} \in \mathbb{T}^{n}$ and let $c_{j}=\sum_{k \geq j} a_{k}$ for $j=$ $1, \ldots, n$. Then

$$
\operatorname{Stable}(t)=\sum_{j=1}^{n} x_{1}^{a_{1}} \cdots x_{j-1}^{a_{j-1}}\left(x_{1}, \ldots, x_{j}\right)^{c_{j}}
$$

Proof. We let $I=\sum_{j=1}^{n} x_{1}^{a_{1}} \cdots x_{j-1}^{a_{j-1}}\left(x_{1}, \ldots, x_{j}\right)^{c_{j}}$. By taking $j=n$ we observe that $t$ belongs to $I$. Furthermore, it follows immediately from the definition of a stable ideal that $I$ is contained in Stable $(t)$. The lemma shows that $I$ is stable and the proof is complete.

The next result yields an important characterization of strongly stable ideals. Henceforth, we are going to call linear segment ideals the ideals of the type $\left(x_{1}, x_{2}, \ldots, x_{h}\right)$.

\section{Proposition 3.5 (Characterization of Strongly Stable Ideals)}

Let $I$ be a monomial ideal in $P$. The following conditions are equivalent

a) The ideal $I$ is strongly stable.

b) The ideal $I$ is a sum of ideals which are intersections of powers of linear segment ideals.

Proof. It is clear that powers of linear segment ideals are strongly stable and it is also clear that sums and intersections of strongly stable ideals are strongly stable. On the other hand, let $I=\left(t_{1}, \ldots, t_{s}\right)$ be a strongly stable ideal. Clearly $I=\operatorname{SStable}\left(t_{1}\right)+\cdots+\operatorname{SStable}\left(t_{s}\right)$. To conclude the proof, it suffices to observe that if $t=x_{1}^{\alpha_{1}} x_{2}^{\alpha_{2}} \cdots x_{n}^{\alpha_{n}}$ then $\operatorname{SStable}(t)=\cap_{i=1}^{n}\left(x_{1}, \ldots, x_{i}\right)^{\beta_{i}}$, where $\beta_{i}=\sum_{j=1}^{i} \alpha_{j}$.

Lemma 3.6 Let $I$ be a strongly stable ideal such that $\operatorname{depth}(P / I)=0$. Then $\operatorname{Sat}(I)$ is strongly stable.

Proof. It is easy to see that $\operatorname{Sat}(I)=I: x_{n}^{r}$ for a sufficiently big $r$. Let $t \in I$ be a power product, and let $t^{\prime}$ be a power product which is obtained from $t$ with a chain of elementary moves (see Definition 3.2). Then $t x_{n}^{r} \in I$, and since $t^{\prime} x_{n}^{r}$ is also obtained from $t$ with a chain of elementary moves. we get $t^{\prime} x_{n}^{r} \in I$, hence $t^{\prime} \in \operatorname{Sat}(I)$.

EXAmple 3.7. Consider the following example. Let $I=\left(x, y^{2}\right) \cap\left(x^{2}, y, z\right)$ in $K[x, y, z]$. Then $I=\left(x^{2}, x y, y^{2}, x z\right)$, hence it is strongly stable. In agreement with the lemma its saturation $\left(x, y^{2}\right)$ is strongly stable, but its embedded component is not. However, it is also possible to write $I=\left(x, y^{2}\right) \cap(x, y, z)^{2}$, and now both components are strongly stable. 


\section{Gin and Distractions}

This section contains the main results of the paper. Before stating the main theorem, we fix a bit of notation. We use the symbol $\mathrm{in}_{\mathrm{drl}}$ to mean $\mathrm{in}_{\text {DegRevLex }}$. If $1 \leq m \leq n$, we write $P_{[m]}$ instead of $K\left[x_{1}, \ldots, x_{m-1}\right]$. Moreover, if $\mathcal{L}=\left(L_{i j} \mid i=1, \ldots, n, j \in \mathbb{N}\right)$ is a distraction matrix, and $1 \leq m \leq n$, we use the notation $\mathcal{L}_{[m]}$ to indicate the matrix $\left(\left(L_{i j}\right)_{[m]} \mid i=1, \ldots, m-1, j \in \mathbb{N}\right)$, where $\left(L_{i j}\right)_{[m]}$ is the image of $L_{i j}+\left(x_{m}, \ldots, x_{n}\right)$ in $P_{[m]}$.

It is clear that if $\mathcal{L}$ is a distraction matrix, then $\mathcal{L}_{[m]}$ need not be such. However, things change if $\mathcal{L}$ is a sufficiently generic distraction matrix.

Lemma 4.1 Let $\mathcal{L}=\left(L_{i j} \mid i=1, \ldots, n, j \in \mathbb{N}\right)$ be a sufficiently generic distraction matrix with entries in $P$, and $1 \leq m \leq n$. Then $\mathcal{L}_{[m]}$ is a sufficiently generic distraction matrix with entries in $P_{[m]}$.

Proof. The claim follows directly from Definition 2.7.

Now we are ready to prove the main result.

\section{Theorem 4.2 (Main Theorem)}

Let $\mathcal{L}$ be a sufficiently generic distraction matrix. Then

$$
\operatorname{in}_{\mathrm{drl}}\left(D_{\mathcal{L}}(I)\right)=I
$$

for every strongly stable monomial ideal $I$ in $P$.

Proof. The proof is achieved through some intermediate claims.

Claim 1. Let $I_{1}, I_{2}$ be two strongly stable monomial ideals and suppose that the theorem is proved for $I_{1}$ and $I_{2}$. Then the theorem holds true for $I_{1}+I_{2}$.

Proof of Claim 1. We let $I=I_{1}+I_{2}$. From Proposition 2.9.e we get the equality $D_{\mathcal{L}}(I)=D_{\mathcal{L}}\left(I_{1}\right)+D_{\mathcal{L}}\left(I_{2}\right)$. We deduce the following chain of relations

$$
I=I_{1}+I_{2}=\operatorname{in}_{\mathrm{drl}}\left(D_{\mathcal{L}}\left(I_{1}\right)\right)+\operatorname{in}_{\mathrm{drl}}\left(D_{\mathcal{L}}\left(I_{2}\right)\right) \subseteq \operatorname{in}_{\mathrm{drl}}\left(D_{\mathcal{L}}\left(I_{1}\right)+D_{\mathcal{L}}\left(I_{2}\right)\right)
$$

which shows that $I \subseteq \operatorname{in}_{\mathrm{drl}}\left(D_{\mathcal{L}}(I)\right)$. Since the two ideals have the same Hilbert function by Proposition 2.9.d, we may conclude that they coincide, and Claim 1 is proved.

Claim 2. Let $I_{1}, I_{2}$ be two strongly stable monomial ideals and suppose that the theorem is proved for $I_{1}$ and $I_{2}$. Then the theorem holds true for $I_{1} \cap I_{2}$.

Proof of Claim 2. We let $I=I_{1} \cap I_{2}$. From Corollary 2.10.b we get the equality $D_{\mathcal{L}}(I)=D_{\mathcal{L}}\left(I_{1}\right) \cap D_{\mathcal{L}}\left(I_{2}\right)$. We deduce the following chain of relations

$$
I=I_{1} \cap I_{2}=\operatorname{in}_{\mathrm{drl}}\left(D_{\mathcal{L}}\left(I_{1}\right)\right) \cap \operatorname{in}_{\mathrm{drl}}\left(D_{\mathcal{L}}\left(I_{2}\right)\right) \supseteq \operatorname{in}_{\mathrm{drl}}\left(D_{\mathcal{L}}\left(I_{1}\right) \cap D_{\mathcal{L}}\left(I_{2}\right)\right)
$$

We conclude again by invoking the coincidence of the Hilbert function of the two ideals. 
Claim 3. Let $1 \leq m \leq n$, let $I$ be a monomial ideal in $P_{[m]}$, and assume that $\operatorname{in}_{\mathrm{drl}}\left(D_{\mathcal{L}_{[m]}}(I)\right)=I$. Then $\operatorname{in}_{\mathrm{drl}}\left(D_{\mathcal{L}_{[m+1]}}\left(I P_{[m+1]}\right)\right)=I P_{[m+1]}$, where $I P_{[m+1]}$ denotes the extension of $I$ to $P_{[m+1]}$.

Proof of Claim 3. Proposition 1.12.c and the obvious remark that DegRevLex restricted to $\mathbb{T}\left(x_{1}, \ldots, x_{m}\right)$ is of $x_{m}$-DegRev type imply the equality

$$
\operatorname{in}_{\mathrm{drl}}\left(D_{\mathcal{L}_{[m+1]}}\left(I P_{[m+1]}\right)\right)+\left(x_{m}\right)=\operatorname{in}_{\mathrm{drl}}\left(D_{\mathcal{L}_{[m+1]}}\left(I P_{[m+1]}\right)+\left(x_{m}\right)\right)
$$

Then we observe that

$$
\operatorname{in}_{\mathrm{drl}}\left(D_{\mathcal{L}_{[m+1]}}\left(I P_{[m+1]}\right)+\left(x_{m}\right)\right)=\mathrm{in}_{\mathrm{drl}}\left(\left(D_{\mathcal{L}_{[m]}}(I)\right) P_{[m+1]}+\left(x_{m}\right)\right)
$$

since $I$ has all the generators in $P_{[m]}$. We use the same argument as before, and say that

$$
\operatorname{in}_{\mathrm{drl}}\left(\left(D_{\mathcal{L}_{[m+1]}}(I)\right) P_{[m+1]}+\left(x_{m}\right)\right)=\mathrm{in}_{\mathrm{drl}}\left(\left(D_{\mathcal{L}_{[m+1]}}(I)\right) P_{[m+1]}\right)+\left(x_{m}\right)
$$

and

$$
\operatorname{in}_{\mathrm{drl}}\left(\left(D_{\mathcal{L}_{[m+1]}}(I)\right) P_{[m+1]}\right)+\left(x_{m}\right)=I P_{[m+1]}+\left(x_{m}\right)
$$

by assumption. Looking at the chain of equalities, we get

$$
\operatorname{in}_{\mathrm{drl}}\left(D_{\mathcal{L}_{[m+1]}}\left(I P_{[m+1]}\right)\right)+\left(x_{m}\right)=I P_{[m+1]}+\left(x_{m}\right)
$$

and hence $I P_{[m+1]} \subseteq \operatorname{in}_{\mathrm{drl}}\left(D_{\mathcal{L}_{[m+1]}}\left(I P_{[m+1]}\right)\right)$. Since both have the same Hilbert function by Corollary 2.10.c, it follows that they coincide, and Claim 3 is proved.

To finish the proof of the theorem, we observe that $I$ can be expressed as a finite combination of sums and intersections of powers of linear segment ideals (see Proposition 3.5). Therefore, after Claims 1 and 2 it suffices to prove the theorem for ideals of the type $\left(x_{1}, \ldots, x_{m}\right)^{s}$. Let us do it. Consider the ring $P_{[m+1]}$, and consider the ideal $I=\left(x_{1}, \ldots, x_{m}\right)^{s}$ in $P_{[m+1]}$. Such ideal coincides with $P_{[m+1]}$ from degree $s$ on. On the other hand the assumption that $\mathcal{L}$ is a sufficiently generic distraction matrix guarantees that its restriction $\mathcal{L}_{[m+1]}$ is also a distraction matrix by Lemma 4 .1. So $D_{\mathcal{L}_{[m+1]}}$ also coincides with $P_{[m+1]}$ from degree $s$ on, by Proposition 2.9.b. And then we get

$$
\operatorname{in}_{\mathrm{drl}}\left(D_{\mathcal{L}_{[m+1]}}(I)\right)=I
$$

We use Claim 3 to extend the above equality to the equality

$$
\operatorname{in}_{\mathrm{drl}}\left(D_{\mathcal{L}}(I P)\right)=I P
$$

The proof is now complete.

The caterpillar is ready to become a butterfly: we are now in a position to tackle the conjecture described in the introduction and made so very plausible by the enormous bulk of corroborative computational evidence. 
Theorem 4.3 Let $I$ be a strongly stable monomial ideal in $P$, and let $\mathcal{L}$ be a distraction matrix. Then

$$
\operatorname{gin}_{\mathrm{drl}}\left(D_{\mathcal{L}}(I)\right)=I
$$

Proof. We observe that $\operatorname{gin}_{\mathrm{drl}}\left(D_{\mathcal{L}}(I)\right)=\operatorname{in}_{\mathrm{drl}}\left(\mathfrak{g}\left(D_{\mathcal{L}}(I)\right)\right)$ where $\mathfrak{g} \in \operatorname{GL}(n, K)$ is generic. Now we use Proposition 2.8.b to get $\operatorname{in}_{\mathrm{drl}}\left(\mathfrak{g}\left(D_{\mathcal{L}}(I)\right)\right)=\mathrm{in}_{\mathrm{drl}}\left(D_{\mathfrak{g} \cdot \mathcal{L}}(I)\right)$, and Proposition 2.8.c to know that $\mathfrak{g} \cdot \mathcal{L}$ is sufficiently generic. A direct application of the Main Theorem 4.2 finishes the proof.

The above formula might suggest that $\operatorname{gin}_{\mathrm{drl}}\left(D_{\mathcal{L}}(I)\right)=\operatorname{gin}_{\mathrm{drl}}(I)$ for every monomial ideal. This is not true even for stable ideals (see the second part of Example 4.6). However, it is true for special monomial ideals which include principal stable ideals, as we are going to show.

Lemma 4.4 Let $r \leq n$, let $t_{1}, \ldots, t_{r} \in \mathbb{T}^{n}$, let $\alpha_{1}, \ldots, \alpha_{r}$ be non-negative integers, and let $I$ be the ideal $\sum_{j=1}^{r} t_{j}\left(x_{1}, \ldots, x_{j}\right)^{\alpha_{j}}$. Assume that $t_{1}=1$, $\mathrm{m}\left(t_{j}\right)<j$ for every $j=2, \ldots, r$, that $t_{j} \mid t_{j+1}$ for $1 \leq j<r$, and that $\operatorname{deg}\left(t_{j}\right)+$ $\alpha_{j} \leq \operatorname{deg}\left(t_{j+1}\right)+\alpha_{j+1}$ for all $1 \leq j<r$.

a) We have $\operatorname{gin}_{\sigma}(I)=\sum_{j=1}^{r} x_{1}^{\operatorname{deg}\left(t_{j}\right)}\left(x_{1}, \ldots, x_{j}\right)^{\alpha_{j}}$ for every term ordering $\sigma$ and for every base field $K$.

b) We have $\operatorname{in}_{\mathrm{drl}}\left(D_{\mathcal{L}}(I)\right)=\operatorname{gin}_{\mathrm{drl}}(I)$ for every sufficiently generic distraction $\mathcal{L}$.

c) We have $\operatorname{gin}_{\mathrm{drl}}\left(D_{\mathcal{L}}(I)\right)=\operatorname{gin}_{\mathrm{drl}}(I)$ for every distraction $\mathcal{L}$

Proof. We let $\beta_{j}=\operatorname{deg}\left(t_{j}\right)$ for $j=1, \ldots, r, I_{j}=t_{j}\left(x_{1}, \ldots, x_{j}\right)^{\alpha_{j}}, J_{j}=$ $x_{1}^{\beta_{j}}\left(x_{1}, \ldots, x_{j}\right)^{\alpha_{j}}$, and $J=\sum_{j=1}^{r} J_{j}$.

To prove a), we observe that $J$ satisfies the assumptions of Lemma 3.3. Moreover, $J$ and $I$ share the same tuple $\left(\left(\operatorname{deg}\left(t_{1}\right), \alpha_{1}\right), \ldots,\left(\operatorname{deg}\left(t_{r}\right), \alpha_{r}\right)\right)$, and hence they share the same Hilbert function by Lemma 3.3.b. Knowing that, to prove the equality $\operatorname{gin}_{\sigma}(I)=J$ it is enough to prove one inclusion, and we will show that $\operatorname{gin}_{\sigma}(I) \supseteq J$. Namely, from $I=\sum_{j} I_{j}$ we get $\operatorname{gin}_{\sigma}(I) \supseteq \sum_{j} \operatorname{gin}_{\sigma}\left(I_{j}\right)$ and $\operatorname{gin}_{\sigma}\left(I_{j}\right)=x_{1}^{\beta_{j}} \operatorname{gin}_{\sigma}\left(\left(x_{1}, \ldots, x_{j}\right)^{\alpha_{j}}\right)=J_{j}$, where the last equality follows from the fact that $\left(x_{1}, \ldots, x_{j}\right)^{\alpha_{j}}$ is strongly stable. The proof of a) is now complete.

To prove b), we denote by $\mathcal{L}_{j}$ the distraction obtained from $\mathcal{L}$ by shifting with $t_{j}$, i.e. $D_{\mathcal{L}_{j}}(t)=D_{\mathcal{L}}\left(t_{j} t\right) / D_{\mathcal{L}}\left(t_{j}\right)$. Then we have the equality $D_{\mathcal{L}}\left(I_{j}\right)=$ $D_{\mathcal{L}}\left(m_{j}\right) D_{L_{j}}\left(\left(x_{1}, \ldots, x_{j}\right)^{\alpha_{j}}\right)$, and hence

$$
\operatorname{in}_{\mathrm{drl}}\left(D_{\mathcal{L}}(I)\right) \supseteq \sum_{j=1}^{r} \operatorname{in}_{\mathrm{drl}}\left(D_{\mathcal{L}}\left(I_{j}\right)\right)=\sum_{j=1}^{r} x_{1}^{\beta_{j}} \operatorname{in}_{\mathrm{drl}}\left(D_{\mathcal{L}_{j}}\left(\left(x_{1}, \ldots, x_{j}\right)^{\alpha_{j}}\right)\right.
$$

Moreover, we get $\operatorname{in}_{\mathrm{drl}}\left(D_{\mathcal{L}_{j}}\left(\left(x_{1}, \ldots, x_{j}\right)^{\alpha_{j}}\right)=\left(x_{1}, \ldots, x_{j}\right)^{\alpha_{j}}\right.$ as a consequence of Theorem 4.3. Summing up, we have shown that $\operatorname{in}_{\mathrm{drl}}\left(D_{\mathcal{L}}(I)\right) \supseteq J$ and we conclude that $\operatorname{in}_{\mathrm{drl}}\left(D_{\mathcal{L}}(I)\right)=J$ since the two ideals have the same Hilbert function.

Claim c) follows from b) by Theorem 4.2. 
We are ready to prove the promised result about principal stable ideals.

Theorem 4.5 Let $t=x_{1}^{a_{1}} \cdots x_{n}^{a_{n}} \in \mathbb{T}^{n}$ and $I=\operatorname{Stable}(t)$. Let $b_{j}=\sum_{k<j} a_{k}$ for $j=1, \ldots, n$, and $c_{j}=\sum_{k \geq j} a_{k}$.

a) We have $\operatorname{gin}_{\tau}(I)=\sum_{j} x_{1}^{b_{j}}\left(x_{1}, \ldots, x_{j}\right)^{c_{j}}$ for every term ordering $\tau$ and every base field $K$.

b) We have $\operatorname{gin}_{\mathrm{drl}}\left(D_{\mathcal{L}}(I)\right)=\operatorname{gin}_{\mathrm{drl}}(I)$ for every distraction $\mathcal{L}$.

Proof. It suffices to combine Proposition 3.4 and 4.4.

EXAMPLE 4.6. The following examples show that the above theorem cannot be generalized to stable monomial ideals (see the warning given after Example 1.14).

In the polynomial ring $K\left[x_{1}, x_{2}, x_{3}, x_{4}\right]$, consider the following ideal $I=$ Stable $\left(\left\{x_{1} x_{2}, x_{2} x_{3} x_{4}\right\}\right)=\left(x_{1}^{2}, x_{1} x_{2}, x_{2}^{3}, x_{2}^{2} x_{3}, x_{2} x_{3}^{2}, x_{2} x_{3} x_{4}\right)$. One can check (with CoCoA) that

$$
\begin{gathered}
\operatorname{gin}_{\mathrm{drl}}(I)=\left(x_{1}^{2}, x_{1} x_{2}, x_{2}^{3}, x_{2}^{2} x_{3}, x_{1} x_{3}^{2}, x_{2}^{2} x_{4}\right) \\
\operatorname{gin}_{\text {lex }}(I)=\left(x_{1}^{2}, x_{1} x_{2}, x_{2}^{3}, x_{2}^{2} x_{3}, x_{1} x_{3}^{2}, x_{1} x_{3} x_{4}\right)
\end{gathered}
$$

Let $I=\operatorname{Stable}\left(\left\{x_{2}^{3}, x_{3}^{2} x_{4}^{2}\right\}\right)$, and $\mathcal{L}$ a generic distraction. Then

$$
\begin{aligned}
& I=\left(x_{1}^{3}, x_{1}^{2} x_{2}, x_{1} x_{2}^{2}, x_{2}^{3},\right. \\
& \left.x_{1}^{2} x_{3}^{2}, x_{1} x_{2} x_{3}^{2}, x_{1} x_{3}^{3}, x_{1} x_{3}^{2} x_{4}, x_{2}^{2} x_{3}^{2}, x_{2} x_{3}^{3}, x_{2} x_{3}^{2} x_{4}, x_{3}^{4}, x_{3}^{3} x_{4}, x_{3}^{2} x_{4}^{2}\right) \\
& \operatorname{gin}_{\mathrm{drl}}(I)=\left(x_{1}^{3}, x_{1}^{2} x_{2}, x_{1} x_{2}^{2}, x_{2}^{3},\right. \\
& \left.x_{1}^{2} x_{3}^{2}, x_{1}^{2} x_{3} x_{4}, x_{1}^{2} x_{4}^{2}, x_{1} x_{2} x_{3}^{2}, x_{1} x_{2} x_{3} x_{4}, x_{1} x_{3}^{3}, \underline{x_{1} x_{3}^{2} x_{4}}, x_{2}^{2} x_{3}^{2}, x_{2} x_{3}^{3}, x_{3}^{4}\right) \\
& \operatorname{gin}_{\mathrm{drl}}\left(D_{\mathcal{L}}(I)\right)=\left(x_{1}^{3}, x_{1}^{2} x_{2}, x_{1} x_{2}^{2}, x_{2}^{3},\right. \\
& \left.x_{1}^{2} x_{3}^{2}, x_{1}^{2} x_{3} x_{4}, x_{1}^{2} x_{4}^{2}, x_{1} x_{2} x_{3}^{2}, x_{1} x_{2} x_{3} x_{4}, x_{1} x_{3}^{3}, x_{2}^{2} x_{3} x_{4}, x_{2}^{2} x_{3}^{2}, x_{2} x_{3}^{3}, x_{3}^{4}\right)
\end{aligned}
$$

We observe that if the minimal generators of a strongly stable monomial ideal $I$ have a non-trivial gcd, then it has to be a pure power of $x_{1}$. So we can generalize Main Theorem 4.2 a bit.

Corollary 4.7 Let $I$ be a strongly stable monomial ideal in $P$, and let $\mathcal{L}$ be a distraction matrix. Let $x_{1}^{a}$ be a non-trivial gcd of the minimal generators of $I$, let $I=x_{1}^{a} J$, and let $F$ be a non-zero form of degree $a$.

a) The monomial ideal $J$ is strongly stable.

b) We have $\operatorname{gin}_{\mathrm{drl}}\left(F D_{\mathcal{L}}(J)\right)=I=x_{1}^{a} J$. 
Proof. Claim a) follows directly from the definition of strongly stable ideal. To prove b), we observe that after a generic change of coordinates, $\operatorname{in}_{\mathrm{drl}}(\mathfrak{g}(F))=x_{1}^{a}$. We argue as in the proof of Theorem 4.3, and get

$$
\begin{aligned}
\operatorname{gin}_{\mathrm{drl}}\left(F D_{\mathcal{L}}(J)\right) & =\operatorname{in}_{\mathrm{drl}}\left(\mathfrak{g}\left(F D_{\mathcal{L}}(J)\right)\right)=\operatorname{in}_{\mathrm{drl}}\left(\mathfrak{g}(F)\left(D_{\mathfrak{g} \cdot \mathcal{L}}(J)\right)\right) \\
& =x_{1}^{a} \operatorname{in}_{\mathrm{drl}}\left(D_{\mathfrak{g} \cdot \mathcal{L}}(J)\right)=x_{1}^{a} J
\end{aligned}
$$

where the last equality follows directly from Theorem 4.3 applied to $J$.

Next result compares the class of all $\operatorname{gin}_{\mathrm{drl}}(I)$ with the class of $\operatorname{gin}_{\mathrm{drl}}(I)$ where $I$ is a radical ideal.

\section{Corollary 4.8 (Gin and Radical Ideals)}

Let $I$ be a homogeneous ideal in $P$. Assume that $\operatorname{depth}(P / I)>0$ and that either $\operatorname{char}(K)=0$ or $\operatorname{char}(K)>0$ and $\operatorname{gin}_{\mathrm{drl}}(I)$ is strongly stable. Then there exists a homogeneous radical ideal $J$ in $P$ such that $\operatorname{gin}_{\mathrm{drl}}(I)=\operatorname{gin}_{\mathrm{drl}}(J)$.

Proof. We let $\mathcal{L}$ be a generic $N$-distraction matrix with a sufficiently big $N$. It is known that $\operatorname{depth}(P / I)=\operatorname{depth}\left(P / \operatorname{gin}_{\mathrm{drl}}(I)\right)$. Proposition 2.26 shows that $D_{\mathcal{L}}\left(\operatorname{gin}_{\mathrm{drl}}(I)\right)$ is radical, and if $J=D_{\mathcal{L}}\left(\operatorname{gin}_{\mathrm{drl}}(I)\right)$ we conclude the proof of Claim a) by Theorem 4.3. Finally, Claim b) is a consequence of a) and Proposition 1.5 .

What happens if we drop the assumption that $\operatorname{depth}(P / I)>0$ ? Clearly, we may get around the difficulty by embedding our ideal into a polynomial ring $\bar{P}$ with one more indeterminate, and then apply Corollary 4.8 to $I$ considered as an ideal in $\bar{P}$. The situation is slightly more complicated if we do not want to extend $P$.

\section{Corollary 4.9 (Gin and Saturation)}

Let $I$ be a homogeneous ideal in $P$, and assume that either $\operatorname{char}(K)=0$ or $\operatorname{char}(K)>0$ and $\operatorname{gin}_{\mathrm{drl}}(I)$ is strongly stable.

a) There exists a homogeneous saturated radical ideal $J$ in $P$ with the property that $\operatorname{Sat}\left(\operatorname{gin}_{\mathrm{drl}}(I)\right)=\operatorname{gin}_{\mathrm{drl}}(J)$.

b) There exists a homogeneous saturated radical ideal $J$ in $P$ with the property that $\operatorname{Proj}\left(P / \operatorname{gin}_{\mathrm{drl}}(I)\right)=\operatorname{Proj}\left(P / \operatorname{gin}_{\mathrm{drl}}(J)\right)$.

Proof. Clearly Claim b) is another way of formulating Claim a). In order to prove Claim a) we distinguish two cases. If $\operatorname{depth}(P / I)>0$, then also $\operatorname{depth}\left(P / \operatorname{gin}_{\mathrm{drl}}(I)\right)>0$, and we conclude the proof by taking $J=D_{\mathcal{L}}\left(\operatorname{gin}_{\mathrm{drl}}(I)\right)$ and invoking Corollary 4.8.a.

Now let $\operatorname{depth}(P / I)=0$, so that also $\operatorname{depth}\left(P / \operatorname{gin}_{\mathrm{drl}}(I)\right)=0$. In this case let $\operatorname{gin}_{\mathrm{drl}}(I)=I^{\prime} \cap Q$ be a decomposition of the ideal $\operatorname{gin}_{\mathrm{drl}}(I)$ so that $\operatorname{depth}\left(P / I^{\prime}\right)>0, \sqrt{Q}=\left(x_{1}, \ldots, x_{n}\right)$, and hence $\operatorname{Sat}\left(\operatorname{gin}_{\mathrm{drl}}(I)\right)=I^{\prime}$.

We let $\mathcal{L}$ be a generic $N$-distraction matrix with a sufficiently big $N$.

Claim 1. The ideal $D_{\mathcal{L}}\left(I^{\prime}\right)$ is radical and is the saturation of $D_{\mathcal{L}}\left(\operatorname{gin}_{\mathrm{drl}}(I)\right)$. 
Proof of Claim 1. The fact that $D_{\mathcal{L}}\left(I^{\prime}\right)$ is radical follows from Proposition 2.26. The fact that it is the saturation of $D_{\mathcal{L}}\left(\operatorname{gin}_{\mathrm{drl}}(I)\right)$ follows from Corollary 2.11.

Claim 2. We have $I^{\prime}=\operatorname{gin}_{\mathrm{drl}}\left(D_{\mathcal{L}}\left(I^{\prime}\right)\right)$.

Proof of Claim 2. It suffices to use Lemma 3.6 to deduce that $I^{\prime}$ is strongly stable, and then invoke Theorem 4.3.

We pick $J=D_{\mathcal{L}}\left(I^{\prime}\right)$, and observe that $J$ is radical by Proposition 2.26 and saturated by Claim 1 . Now Sat $\left(\operatorname{gin}_{\mathrm{drl}}(I)\right)=I^{\prime}$, and $I^{\prime}=\operatorname{gin}_{\mathrm{drl}}(J)$ by Claim 2 , so that the proof is complete.

As another consequence, we get the following corollary. This result brings finite sets of special points in projective space into the spotlight.

\section{Corollary 4.10 (Gin and Points)}

Let $I$ be a zero-dimensional strongly stable monomial ideal in $P$, and let $\mathcal{L}$ be a distraction matrix which is radical for $I$, and whose entries are in the polynomial ring $\bar{P}=K\left[x_{1}, \ldots, x_{n}, x_{n+1}\right]$. Then $D_{\mathcal{L}}(I)$ is the ideal of a finite set of rational points in $\mathbb{P}_{K}^{n}$ such that $\operatorname{gin}_{\mathrm{drl}}\left(D_{\mathcal{L}}(I)\right)=I \bar{P}$.

Proof. The equality $\operatorname{gin}_{\mathrm{drl}}\left(D_{\mathcal{L}}(I)\right)=I \bar{P}$ follows directly from Theorem 4.3. So we have only to show that $D_{\mathcal{L}}(I)$ is the ideal of a finite set of rational points in $\mathbb{P}_{K}^{n}$. We have

$$
\operatorname{dim}\left(\bar{P} / D_{\mathcal{L}}(I)\right)=\operatorname{dim}\left(\bar{P} /\left(\operatorname{gin}_{\mathrm{drl}}\left(D_{\mathcal{L}}(I)\right)\right)=\operatorname{dim}(\bar{P} / I)=\operatorname{dim}(P / I)+1=1\right.
$$

The assumption on $I$ and Proposition 2.26 imply that $D_{\mathcal{L}}(I)$ is radical, hence $D_{\mathcal{L}}(I)$ defines a radical zero-dimensional scheme in $\mathbb{P}_{K}^{n}$, i.e. a scheme of points in $\mathbb{P}_{K}^{n}$. They are rational, since their defining ideals are linear by Lemma 2.24.a, and the proof is complete.

For example, if we are given a zero-dimensional strongly stable monomial ideal $I$ in $K\left[x_{1}, \ldots, x_{n}\right]$, we embed it in $K\left[x_{1}, \ldots, x_{n}, x_{n+1}\right]$. Then we apply either the classic or the generic distraction with $N$ sufficiently large, and get an ideal in $K\left[x_{1}, \ldots, x_{n}, x_{n+1}\right]$ which defines a finite set of rational points in $\mathbb{P}^{n}$, and whose $\operatorname{gin}_{\mathrm{drl}}$ is $I$ itself. 


\section{References}

[E95] D. Eisenbud, Commutative Algebra with a view Toward Algebraic Geometry, Springer Verlag, 1995.

[EK90] S. Eliahou, M. Kervaire, Minimal resolutions of some monomial ideals, J. Algebra 129 (1990), no. 1, 1-25.

[G96] M. L. Green, Generic Initial Ideals, Centre de Recerca Matemàtica, Summer School on Commutative Algebra Vol 2/7 (1996), 11-76.

[H66] R. Hartshorne, Connectedness of the Hilbert scheme, Inst. Hautes Etudes Sci. Publ. Math. no. 29 (1966), 5-48.

[KR00] M. Kreuzer and L. Robbiano, Computational Commutative Algebra 1, Springer Verlag, 2000.

[KR04] M. Kreuzer and L. Robbiano, Computational Commutative Algebra 2, Springer Verlag, In preparation.

[MN00] J. Migliore, U. Nagel, Lifting monomial ideals, Special issue in honor of Robin Hartshorne. Comm. Algebra 28 (2000), no. 12, $5679-5701$.

[P94] K. Pardue, Non-standard Borel-fixed ideals, PhD Thesis, Brandeis University, May 1994.

[P96] K. Pardue, Deformation classes of graded modules and maximal Betti numbers, Illinois J. Math. 40 (1996), no. 4, 564-585.

Address of the authors:

Dipartimento di Matematica, Università di Genova, Via Dodecaneso 35, I-16146 Italy.

E-mail: bigatti,conca, robbiano@dima.unige.it 